\title{
Hybrid Energy Efficient Routing Protocol for Wireless Sensor Networks
}

\section{Fazel Farazandeh}

\author{
Submitted to the \\ Institute of Graduate Studies and Research \\ in partial fulfillment of the requirements for the Degree of
}

Master of Science

in

Electrical and Electronic Engineering

Eastern Mediterranean University

August 2012

Gazimağusa, North Cyprus 
Approval of the Institute of Graduate Studies and Research

\section{Prof. Dr. Elvan Yilmaz \\ Director}

I certify that this thesis satisfies the requirements as a thesis for the degree of Master of Science in Electrical and Electronic Engineering.

Assoc. Prof. Dr. Aykut Hocanın

Chair, Department of Electrical and Electronic

Engineering

We certify that we have read this thesis and that in our opinion it is fully adequate in scope and quality as a thesis for the degree of Master of Science in Electrical and Electronic Engineering.

Prof. Dr. Şener Uysal

Supervisor

Examining Committee

1. Prof. Dr. Şener Uysal

2. Assoc. Prof. Dr. Aykut Hocanın

3. Asst. Prof. Dr. Rasime Uyguroğlu 


\begin{abstract}
Deployment of wireless sensor networks is rapidly increasing in many different monitoring and control applications. Design and implementation of wireless sensor networks, requires diverse knowledge from several different disciplines. Therefore, this is a new interdisciplinary technology, which has been discussed widely in the literatures.
\end{abstract}

Sensor nodes of the WSNs are powered by limited resources, which are mostly the batteries with constrained energy. Design of an energy efficient routing protocol contributes to increasing the network lifetime. In this thesis, based on energy analysis of the routing protocols, a new method of data transmission is proposed. However, design and applying a routing protocol in WSNs is very application specific and can be changed for different parameters of the environment.

The new proposed method is applicable for different network sizes, while the energy efficiency of the protocol is the main characteristics of the design. Moreover, some other important parameters, which affect the efficiency of the transmission, such as number of nodes and massage length, are considered during the simulations.

Keywords: Routing protocols, Energy Efficiency, Wireless Sensor Networks, Energy analysis. 


\section{ÖZ}

Son zamanlarda kablosuz sensor ağlarının birçok değişik uygulamadaki kullanımı hızlı bir şekilde artmaktadır. Kablosuz Sensör Ağlarının tasarımı ve uygulaması farklı alanlarda bilgi sahibi olunmasını gerektirmektedir. Dolaysıyla, bu konu birçok araştırmada incelenen yeni bir disiplinlerarası teknoloji olarak düşünülebilmektedir.

Kablosuz Sensör Ağlarındaki sensör düğümlerinin enerjisi çoğunlukla sınırlı kapasiteye sahip olan pillerden oluşan sınırlı kaynaklar tarafindan temin edilmektedir. Enerji açısından verimli olan bir yönlendirme protokolunun tasarımı ağın ömür süresinin artmasına katkıda bulunmaktadır. Bu tez çalışmasında, yönlendirme protokollarına ait enerji analizleri esas alınarak, yeni bir bilgi aktarma yönteminin geliştirilmesi amaçlanmıştır. Yine de Kablosuz Sensör Ağları konusunda bir yönlendirme protokolunun tasarlanması ve uygulanması yüksek oranda yapılacak olan uygulamaya bağlı ve buna özel olup çeşitli ortam parametreleri için değiştirilebilmektedir.

Geliştirilen yeni yöntemde protokolun enerji verimliliği tasarım sürecinin asıl karakteristiğini oluştururken bu yöntem çeşitli ağ boyutları için uygulanabilmektedir. Bunun dışında düğüm sayıları ve mesaj uzunluğu gibi aktarma süreci üzerinde etkili olan başka parametreler de simülasyon sürecinde dikkate alınmıştır.

Anahtar Kelimeler: Yönlendirme protokolunun, Enerji açısından, Kablosuz Sensör Ağları, Enerji analizleri. 
Dedicated to

My parents, Sisters, Brother

And B.R 


\section{ACKNOWLEDGMENT}

I would like to take this opportunity to thank my supervisor Prof. Dr. Şener Uysal for his support and his patience during my study.

Besides, I would like to thank my dear friend Dr. Reza Abrishambaf, who generously shared his knowledge with me and I am honored of cooperating with him in my thesis.

I am also thankful to all the faculty members at the department of Electrical and Electronic Engineering, and specially the chair, Assoc. Prof. Dr. Aykut Hocanın and vice chair, Assoc. Prof. Dr. Hasan Demirel.

I would like also to express my deepest appreciation to my parents and my siblings who support me in all of my life. 


\section{TABLE OF CONTENTS}

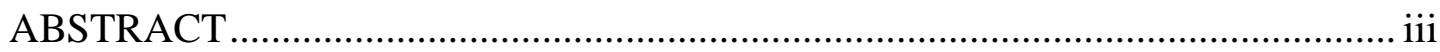

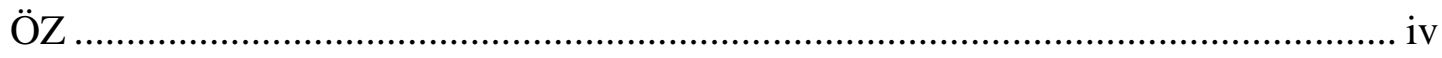

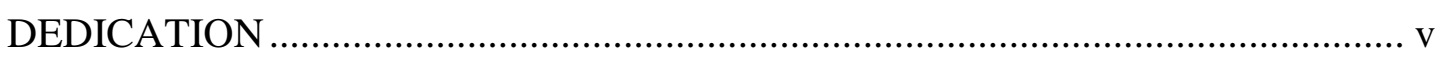

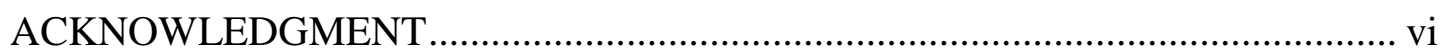

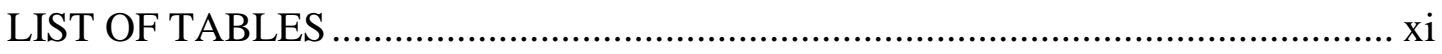

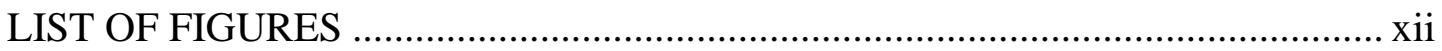

LIST OF SYMBOLS AND ABBREVIATIONS …........................................... xiii

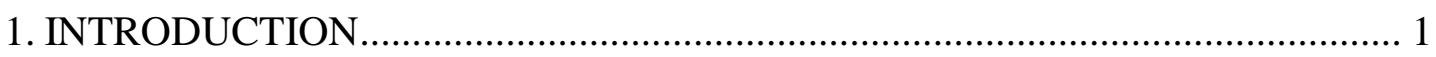

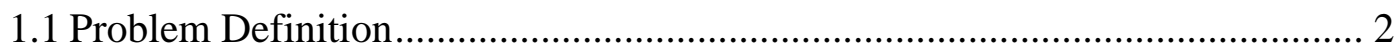

1.2 Outline

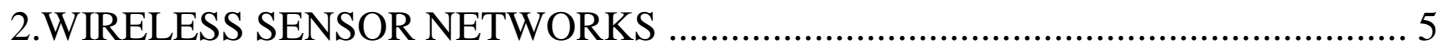

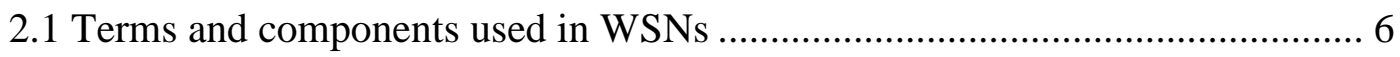

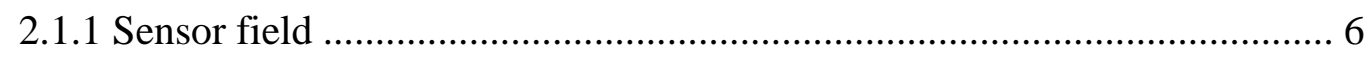

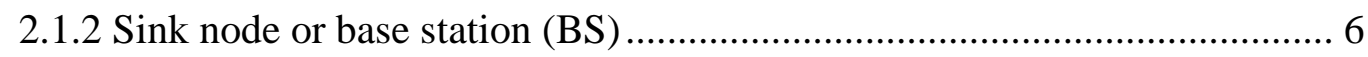

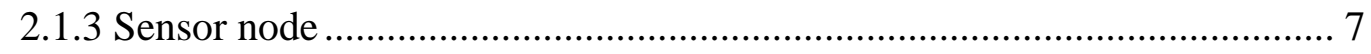

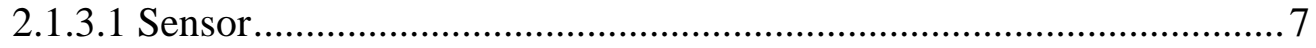

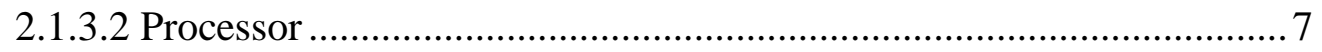

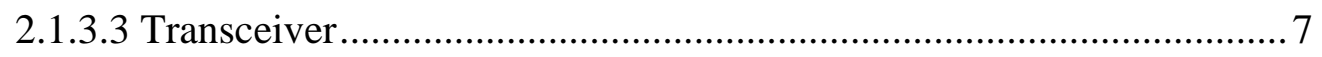

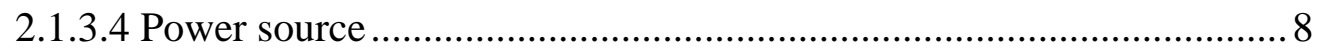

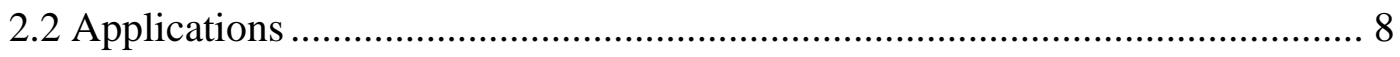

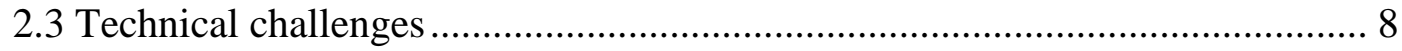

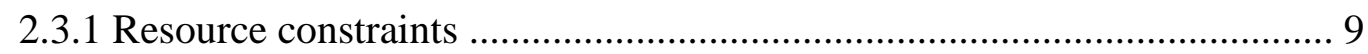

2.3.2 Harsh conditions environment and dynamic topologies .......................... 9 


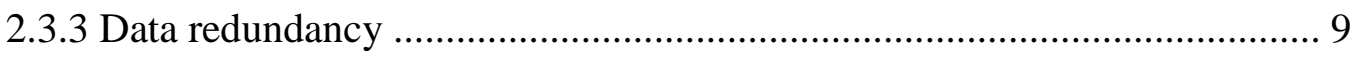

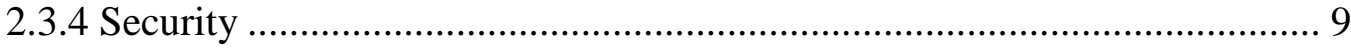

2.3.5 Ad hoc architecture and large size ................................................... 10

2.3.6 Integration with other networks and Internet ........................................ 10

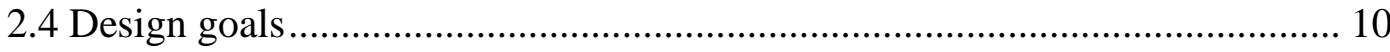

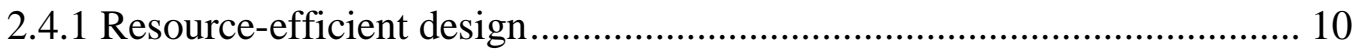

2.4.2 Adaptive network operation ............................................................. 10

2.4.3 Localized processing and data fusion .................................................. 11

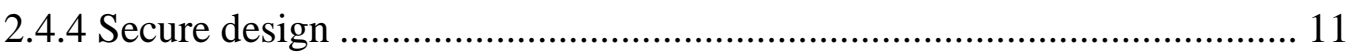

2.4.5 Small size and low-cost sensor nodes ................................................ 11

2.4.6 Efficient protocols and scalable architectures ...................................... 11

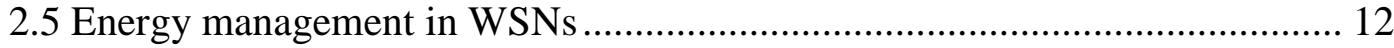

2.5.1 Energy management in data acquisition and transmission .................... 12

2.5.2 Major sources of energy waste in WSNs ............................................ 13

3. ROUTING PROTOCOLS IN WIRELESS SENSOR NETWORKS ................... 14

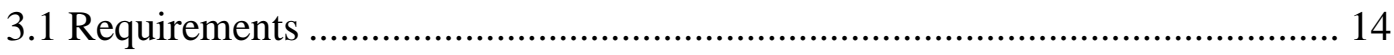

3.1.1 Data-centric communication ............................................................ 14

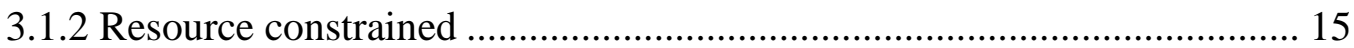

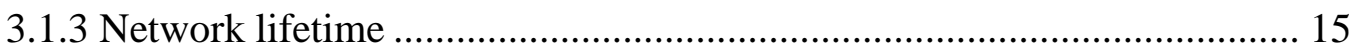

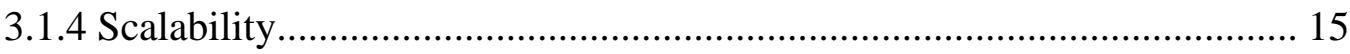

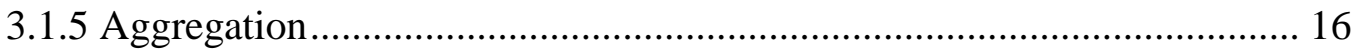

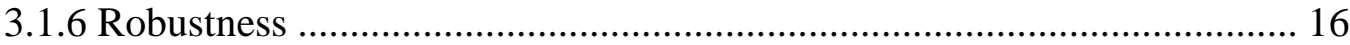

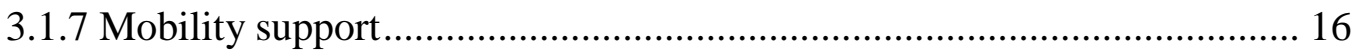

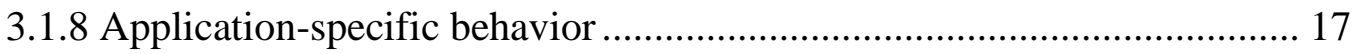

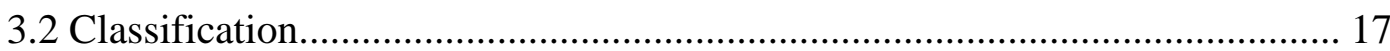


3.2.1.1 Geographic Adaptive Fidelity (GAF) ........................................... 18

3.2.1.2 Geographic and Energy-Aware Routing (GEAR) .......................... 19

3.2.1.3 Minimum Energy Communication Network (MECN) ..................... 19

3.2.2 Data-centric protocols ................................................................... 19

3.2.2.1 Energy-Aware Data-Centric Routing (EAD)..................................20

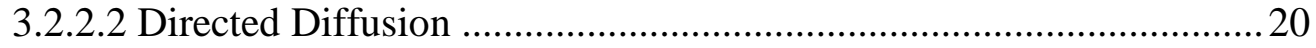

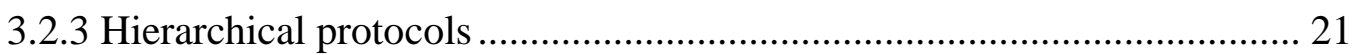

3.2.3.1 Low-energy adaptive clustering hierarchy (LEACH) ...................... 22

3.2.3.2 Power-Efficient Gathering in Sensor Information Systems

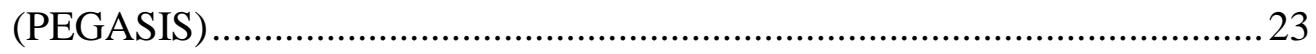

3.2.3.3 Hybrid, Energy-Efficient Distributed Clustering (HEED) ..............23

3.2.4 Mobility-based Protocols ....................................................................... 24

3.2.4.1 Scalable Energy-Efficient Asynchronous Dissemination (SEAD).... 24

3.2.4.2 Dynamic Proxy Tree-Based Data Dissemination ............................ 24

3.2.5 Multipath-based protocols............................................................... 25

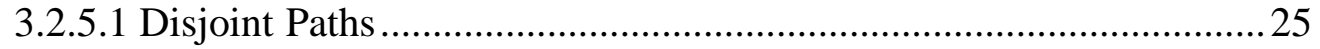

3.2.5.2 Braided Paths................................................................................ 26

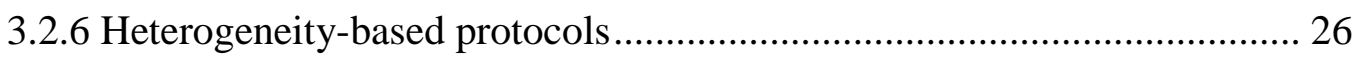

3.2.6.1 Cluster-Head Relay Routing (CHR) ............................................ 26

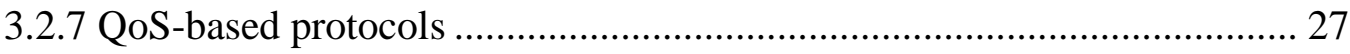

3.2.7.1 Sequential Assignment Routing (SAR) ........................................2

3.2.7.2 Energy-Aware QoS Routing Protocol ........................................... 27

4. ENERGY ANALYSIS OF THE DIRECT AND MINIMUM TRANSMISSION

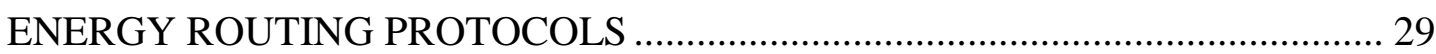


4.1 Energy calculation

4.2 Analysis of routing protocols in WSNs ....................................................... 32

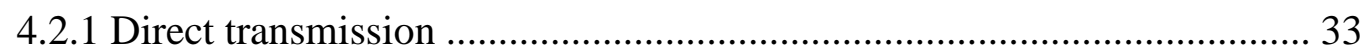

4.2.2 Minimum transmission energy method (MTE) ..................................... 34

4.2.3 Simulation of Direct and MTE routing protocols ................................. 39

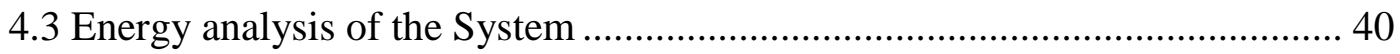

5. HYBRID ENERGY EFFICIENT (HEE) ROUTING PROTOCOL ..................... 47

5.1 Introducing Hybrid Energy Efficient (HEE) routing protocol....................... 47

5.2 Design and algorithm for Hybrid Energy Efficient (HEE) ........................... 47

5.3 HEE versus Direct and MTE methods......................................................... 49

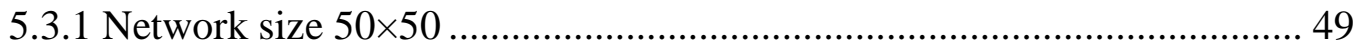

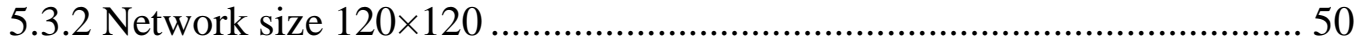

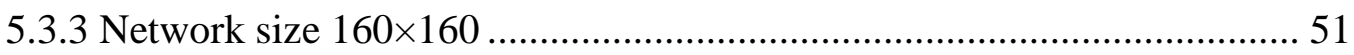

5.4 The effect of massage length and Network size on total energy consumption of the

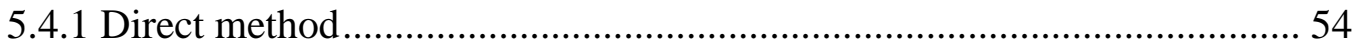

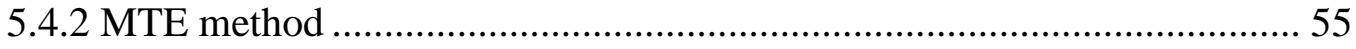

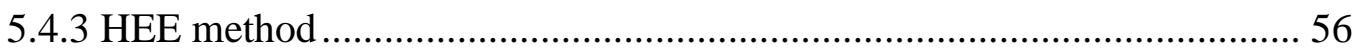

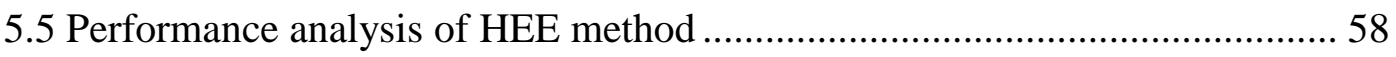

5.5.1 Computational Complexity of HEE ................................................... 59

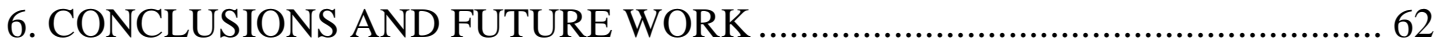

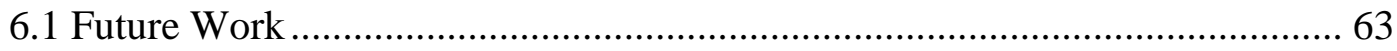

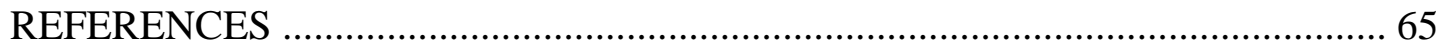




\section{LIST OF TABLES}

Table 2-1: Technical challenges versus design goals in WSNs.............................. 12

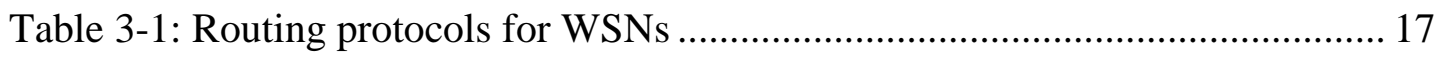

Table 4-1: Different environments and corresponding path loss exponent .............. 30

Table 4-2: dcrossover for different frequencies ............................................... 31

Table 4-3: Total Energy dissipation of the system versus network size.................... 46

Table 5-1: Total Energy dissipation of the system over different network sizes....... 53

Table 5-2: Min, Max, and average values of direct, MTE and HEE methods........... 58

Table 5-3: Computational complexity of Direct, MTE and HEE .................60 


\section{LIST OF FIGURES}

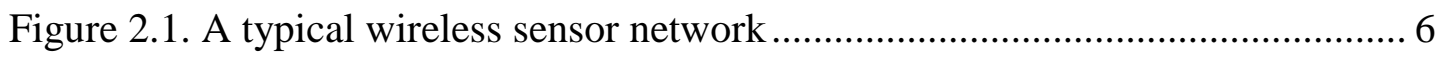

Figure 3.1. State transition diagram of GAF................................................... 18

Figure 3.2. Cluster-based Hierarchical Model .................................................... 21

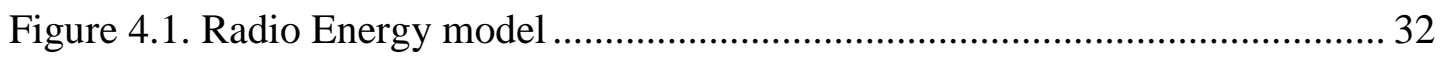

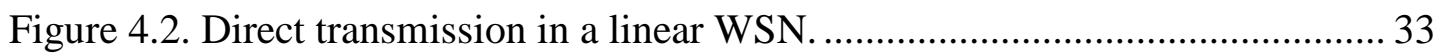

Figure 4.3. Direct transmission in a distributed WSN ........................................... 34

Figure 4.4. Direct minimum transmission energy (MTE) in a linear WSN............... 35

Figure 4.5. Randomly distributed WSN using MTE method ................................. 36

Figure 4.6. Finding path in the MTE routing protocol.......................................... 37

Figure 4.7. Algorithm of path finding in MTE method ........................................... 38

Figure 4.8. Simulation of direct protocol and path finding algorithm in MTE ......... 39

Figure 4.9. All of the nodes acting as source node one by one............................... 41

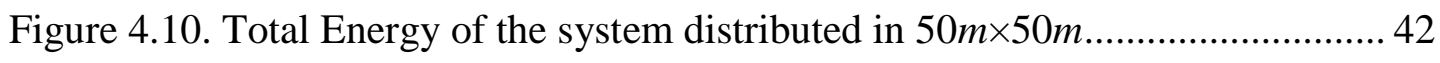

Figure 4.11. Total Energy of the system distributed in $120 \mathrm{~m} \times 120 \mathrm{~m} \ldots \ldots \ldots \ldots \ldots \ldots \ldots \ldots . . . . . . . . . .44$

Figure 4.12. Total Energy of the system distributed in $160 \mathrm{~m} \times 160 \mathrm{~m} \ldots \ldots \ldots \ldots \ldots \ldots \ldots . . . . . . . . . . .45$

Figure 5.1. Algorithm of Hybrid Energy Efficient (HEE) method .......................... 48

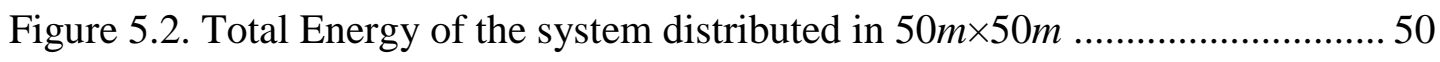

Figure 5.3. Total Energy of the system distributed in $120 \mathrm{~m} \times 120 \mathrm{~m} \ldots \ldots \ldots \ldots \ldots \ldots \ldots . . . . . . . . . . . .51$

Figure 5.4. Total Energy of the system distributed in $160 \mathrm{~m} \times 160 \mathrm{~m} \ldots \ldots \ldots \ldots \ldots \ldots \ldots . . . . . . . . . . . .52$

Figure 5.5. Total energy of the system using direct method .................................. 54

Figure 5.6. Total energy of the system using MTE method …............................... 55

Figure 5.7. Total energy of the system using HEE method ................................... 56

Figure 6.1. Data transmission for one random sensor node using IHEE method ...... 63 


\section{LIST OF SYMBOLS AND ABBREVIATIONS}

\begin{tabular}{|c|c|}
\hline$c$ & Speed of Light \\
\hline$d$ & Distance \\
\hline$d_{\text {critical }}$ & Critical Distance \\
\hline$d_{\text {crossover }}$ & Crossover Distance \\
\hline$E_{\text {elec }}$ & Energy Consumed to process one bit \\
\hline$E_{n \text { to } B(M T E)}$ & Required energy for transmitting $k$ bit data from node $n$ to BS \\
\hline$f_{t}$ & Transmission Frequency \\
\hline$h_{r}$ & Receiver's Height above the Ground \\
\hline$h_{t}$ & Transmitter's Height above the Ground \\
\hline$k$ & Number of bits \\
\hline$L$ & System Loss Factor \\
\hline$n$ & Path Loss Exponent \\
\hline$\epsilon_{a m p}$ & Energy consumed for amplifying station \\
\hline$\lambda$ & Wavelength \\
\hline API & Application Programming Interface \\
\hline $\mathrm{BS}$ & Base Station \\
\hline HEE & Hybrid Energy Efficient \\
\hline $\mathrm{CH}$ & Cluster Head \\
\hline CHR & Cluster-Head Relay Routing \\
\hline EAD & Energy-Aware Data-Centric Routing \\
\hline GAF & Geographic Adaptive Fidelity \\
\hline GEAR & Geographic and Energy-Aware Routing \\
\hline HEED & Hybrid, Energy-Efficient Distributed Clustering \\
\hline
\end{tabular}


HVAC Heating, Ventilation \& Air conditioning

IHEE Intelligent Hybrid Energy Efficient

IP Internet Protocol

LEACH Low-energy adaptive clustering hierarchy

MANET Mobile Ad hoc Network

MECN Minimum Energy Communication Network

MTE Minimum Transmission Energy

PEGASIS Power-Efficient Gathering in Sensor Information Systems

QoS Quality of service

SAR Sequential Assignment Routing

SEAD Scalable Energy-Efficient Asynchronous Dissemination

WSN Wireless Sensor Network 


\section{Chapter 1}

\section{INTRODUCTION}

A wireless sensor network (WSN) consist of several number of tiny sensor nodes from a few to hundreds or even thousands, distributed over a geographical area also termed as sensing field. Each node is a low-power device, which simultaneously has the ability of computing, wireless communication and sensing data [1].

Power source considered as one of the main parts of the sensor nodes. In fact, sensor nodes are generally powered by the batteries, with limited capacity. In WSNs, power consumption mainly happens in three sections: sensing, communication, and data processing. Due to the environmental constraints, most of the times the batteries can neither be replaced or recharged [2]. Therefore, in order to increase the network lifetime, energy of the batteries must be used judiciously.

Routing layer in the wireless sensor networks is a layer, which is responsible for transferring collected data, from each sensor node to the base station. As packet of data can be transmitted through different paths, making decision for selecting the path has an important effect on the energy efficiency of the system. Thus, the energy efficiency of the routing protocols is the key factor of designing a wireless sensor network. 


\subsection{Problem Definition}

There are many researches in which design principals, and technical approaches of routing protocols of WSNs have been discussed. In this thesis, two popular routing protocols, namely direct transmission and minimum energy transmission (MTE) are selected as the basic methods of transmission. These two routing protocols are desirable for many applications of WSNs. However, direct method is more energy efficient for small networks, while MTE has higher quality of performance in largescale areas. Apart from the network size (distance between the nodes), many other characteristics of WSNs such as: Massage length, number of nodes and transmission frequency, have crucial effects on the efficiency of the routing protocols. Considering to above facts brings the idea to the mind, that how would operate the combination of two direct and MTE methods, while the network size, massage length or number of nodes are variable.

\subsection{Outline}

In this research, an energy efficient routing protocol based on two methods namely direct and MTE, will be presented as a new routing protocol and it will be named Hybrid Energy Efficient (HEE) routing protocol.

The procedure of calculating energy dissipation of the new routing protocol is started by choosing one of the sensor nodes as a source or starting point. In this phase, consumed energy using direct method, for transmitting packet of data to the base station is calculated. Then required energy of the MTE method, in order to send the same packet of data from the same source node to the base station, will be computed. So far, required energy of both methods from same source node to the sink is acquired. 
Therefore, by comparing these two values, that one which is more efficient, can be selected as a desired method of transmission for this specific node. In addition, it would be the value, which takes place in the HEE method. This procedure can be applied for the entire nodes, as each of them once selected to act as a source node. It will be continued, until HEE values will be calculated for all sensor nodes. Finally, by adding all values of HEE method, total energy expended over the system will be calculated, when the new Hybrid energy efficient routing protocol is in use.

In this thesis, two above mentioned transmission methods (direct and MTE) are modeled in 2D space using the relevant algorithms. In fact, proposed algorithm for MTE and its simulation in 2D space enable us to calculate energy dissipation of the system, which is one of the main works in this study. Therefor another algorithm is developed for HEE and energy consumption for data transmission by this method will be calculated.

HEE is a method, which can be used in the networks with variable sizes, massage length or number of nodes. This is the most important advantages of deploying HEE as transmission method of the system. Many real case applications in environment monitoring or industrial control may be found for the Hybrid Energy Efficient method.

There are five more chapters in this thesis, as follows: Chapter 2 presents the principals of the WSN along whit some applications and energy management views. Chapter 3 discusses about the popular routing protocols of WSNs, and their classifications. In Chapter 4, two basic methods of this thesis (direct and MTE) are analyzed when they are deployed into the randomly distributed WSNs. Chapter 5, 
introduces the proposed Hybrid energy efficient (HEE) routing protocol, where this new method is compared with direct and MTE methods. Chapter 6 is the conclusion of this thesis. 


\section{Chapter 2}

\section{WIRELESS SENSOR NETWORKS}

A wireless sensor network (WSN) is composed of several number of sensor nodes spatially distributed over a geographical area which is called sensor field. Each node is a device with limited power source, which in the same time has the ability of computing, wireless communication and sensing data [1]. Nodes manage themselves in clusters and networks and cooperate to carry out the assigned task, when there is no human intervention. Sensor nodes are typically able to sense physical environmental information (e.g., temperature, humidity, vibration, acceleration or whatever required), process locally the acquired data both at unit and cluster level, and send the outcome or aggregated data to the cluster and/or one or more collection points, named sinks or base stations (BS).

A WSN can thus be considered as an intelligent distributed measurement technology appropriate for many different monitoring and control circumstances. Recently, the number of sensor network deployments for real-life applications has rapidly increased, with a trend expected to more growth in the incoming years. Figure 2.1 shows a typical wireless sensor network. 


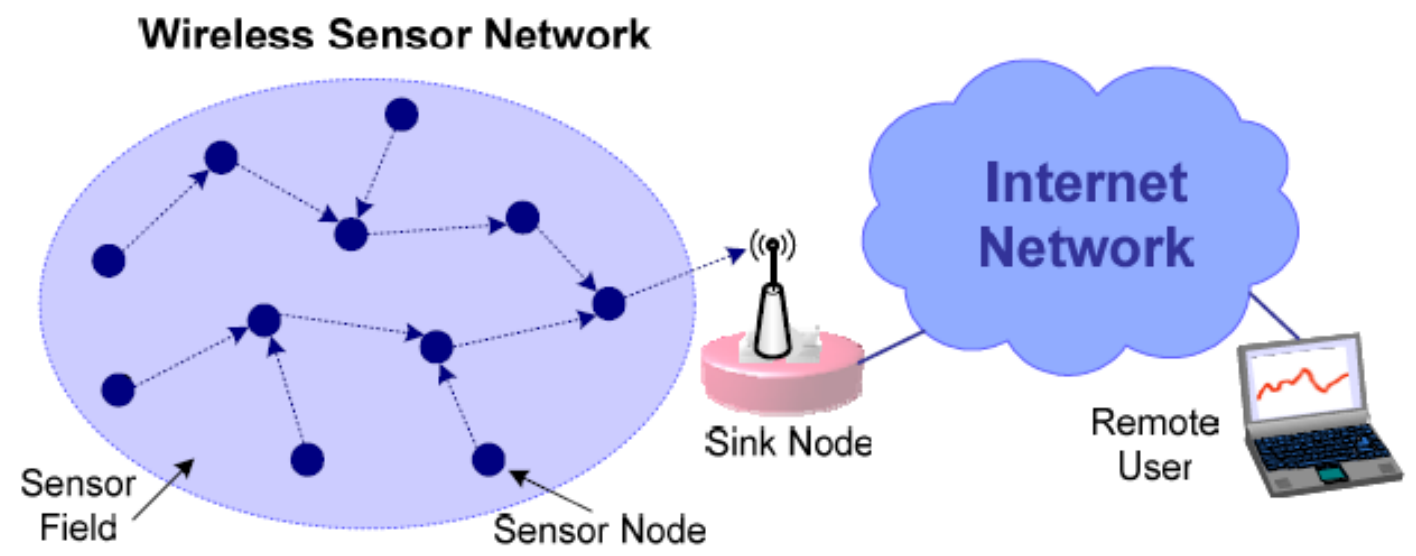

Figure 2.1. A typical wireless sensor network [2]

\subsection{Terms and components used in WSNs}

The most important terms and components which is frequently used in the wireless sensor network technology, are as follows.

\subsubsection{Sensor field}

The sensor field or sensing field is the region in which sensor nodes are spatially distributed over the coverage area. Each node is connected to one or perhaps several sensor nodes and all of them need to be able to connect with the sink node. Each such field is predefined according to the parameters, which is required to be measured, monitored or controlled. Then few to many sensor nodes can be distributed over the field, which is needed to be as coverage area of the network.

\subsubsection{Sink node or base station (BS)}

The base stations are one of the main components of the wireless sensor network. A WSN may have one or more sink node, which operate as a gateway between sensor nodes and the end user. Comparing to the sensor nodes, usually a base station benefits from much more computational, communication and energy resources. 


\subsubsection{Sensor node}

Sensor node is an integrated single chip with the abilities of sensing, processing, data collection and wireless communication. There is also a limited power supply attached to this tiny device. Developing an efficient design in both of hardware and software sections is crucially important, since the sensor nodes are the main components, which determine the efficiency of the WSNs. Typical sensor nodes composed of four basic parts as follows.

\subsubsection{Sensor}

Sensors are the hardware devices, which detect any variation in the physical condition such as pressure, temperature, current, voltage, etc. and produce a measurable response for each change. Analog signals are produced, whenever the sensors observe a phenomenon. By using the analog-to-digital converters, these analog signals will be converted to digital signals. Therefore, data signals will be forwarded to the processor, for more necessary processing.

\subsubsection{Processor}

The tasks such as control of the functionality of other components and data processing are assigned to the processing unit. Processors are often associated with a storage unit by limited size of memory.

\subsubsection{Transceiver}

The node is connected to the network by the transceiver unit. Typically, radios of theses transceivers work in four modes:

1) receive; 2) transmit; 3) sleep; and 4) idle. Power consumption of the radios in the idle mode, is likely equal to the consumed power in receiving mode. Therefore, it is preferred to turn off the radios rather than operating in the idle mode, when it is not receiving or transmitting. 


\subsubsection{Power source}

Power source is one of the main parts of the sensor nodes of WSNs. In fact, sensor nodes are generally powered by the batteries, with limited capacity. In WSNs, power consumption mainly happens in three sections: sensing, communication, and data processing. Due to the environmental constraints, most of the times the batteries can neither be replaced or recharged. Therefore, in order to increase the network lifetime, energy of the batteries must be used judiciously.

\subsection{Applications}

There are two types of applications for WSNs. First type is the traditional sensor network in which wiring is replaced by wireless communication. Moreover, the other type is completely new one. Small size of the sensor nodes and free-maintenance operation of WSNs, enable them to be used in variable environments. Further to local data processing and the integration, many other capabilities of WSNs allow their rapid deployments in completely new types of application. Several physical quantities can be sensed and reported via WSNs. In addition, the miniaturization of sensing devices allows the embedding of small WSN nodes as an unnoticeable part of the everyday life. Home automation, Environmental monitoring, Industrial monitoring and control, Security and Health care can be mentioned as the most typical applications of WSNs [8].

\subsection{Technical challenges}

According to the individual characteristics of the WSNs, in the developing and design of these kinds of networks, combination of expertise from different disciplines such as industrial, sensor-technology, and RF design is required. Moreover, networking expertise play an important role for the understanding and accomplishment of the WSNs. deploying all of the above knowledge brings unique 
challenges to the all specialist of these industrial disciplines. The typical challenges in design and implementation of the WSNs can be listed as follows [3].

\subsubsection{Resource constraints}

Design and implementation of the wireless sensor networks have these three resource constraints: a) energy; b) memory; c) processing. Sensor nodes are limited by the energy of the batteries, and by the physical size. In addition, the memories are constrained and the computational capabilities are limited.

\subsubsection{Harsh conditions environment and dynamic topologies}

According to the link or node-failure, connectivity and topology of the network can be changed. Moreover, some conditions such as: FR interface, high level of humidity, dust and dirt may happen to the sensor nodes of the networks, which bring challenges to the performance. These harsh environment situations and dynamic topology of the network, probably cause to number of nodes to do not functioning properly.

\subsubsection{Data redundancy}

According to the high density, observation of the sensors is extremely correlated in the space domain. Moreover, the character of the phenomenon which is mostly physical, may forms the temporal correlation between each successive monitoring of sensor node.

\subsubsection{Security}

Intrusion and external denial-of-service (DoS) attacks are the possible threats to the WSNs. Hence, the security needs to be considered as a crucial feature of the WSNs. New designed WSNs equipped with the new technologies for the security attacks. There are two types of threatening for the security of WSNs namely passive and active attacks. 


\subsubsection{Ad hoc architecture and large size}

In many cases wireless sensor networks consist of large number of nodes (hundreds to thousands or more), which is randomly distributed through the large-scale field.

\subsubsection{Integration with other networks and Internet}

This is important for WSNs to supply services that make the querying of the network enable to retrieve convenient information at whatever time and from anywhere. For this purpose, the wireless sensor networks must be accessible remotely via Internet, and also should be integrated with Internet Protocol (IP) architecture. Currently WSNs use gateway as an intermediate device in order to connecting to the internet. However, it is not so far in the future that sensor nodes will be able to be integrated to the internet directly.

\subsection{Design goals}

The current wireless sensor networks span a wide range of utilizations, including all of the aforementioned applications in the previous section. In order to meet the different requirement of these applications and dealing with the technical challenges, below design goals have to be followed:

\subsubsection{Resource-efficient design}

In order to provide maximum lifetime for the wireless sensor networks, energy efficiency is the key fact. Conservation of the energy may be considered in all of components of the WSNs. Energy efficient protocols can be deployed to the WSNs. In the network layer, energy aware routing protocol and in the MAC layer, energy saving mode are the examples of efficient design.

\subsubsection{Adaptive network operation}

In WSNs, adaptability is significantly important, since this feature allows end-users to adapt dynamic or varying wireless-channel states in different environments and 
requirements of new connection. For balancing the tradeoffs between accuracy, latency, resources, and time-synchronization requirements, communication protocols and adaptive signal-processing algorithms are needed.

\subsubsection{Localized processing and data fusion}

Based on the application requirement, sensor nodes are able to filter the data locally. Therefore, instead of transmitting the raw data to the base station, only processed data will be sent to the sink. Using this characteristic, the overhead of the system is decreased, while only the necessary information is forwarded to the BS.

\subsubsection{Secure design}

In the design of the security mechanism for the wireless sensor networks, both of the low-level and high-level security basics must be considered [5]. Moreover, according to resource limitation, the overhead of the security method must be reasonable when it is compared to the other requirement of the QoS performance.

\subsubsection{Small size and low-cost sensor nodes}

Low-cost and Compact sensor nodes are very crucial to fulfill the large-scale deployment of wireless sensor networks. It should be reminded that the owner of the system have to think about the ownership cost including (modifications, packaging requirements, maintainability, etc.), replacement, costs of logistics and implementation, and servicing costs, along with the per unit price all of them together.

\subsubsection{Efficient protocols and scalable architectures}

As the WSNs support diverse applications with large number of requirements, developing flexible and scalable architectures is essential. Modular and hierarchical systems can increase the system flexibility, robustness, and reliability. In addition, 
efficient protocols enhance the operation efficiency of the sensor nodes and network lifetime.

Table 2-1 summarizes the challenges of WSNs and the corresponding design goals for each of them.

Table 2-1: Technical challenges and design goals of wireless sensor networks [3]

\begin{tabular}{|c|c|}
\hline Challenges & Design Goals \\
\hline Resource constraints & Resource efficient design \\
\hline $\begin{array}{c}\text { Dynamic topologies and harsh } \\
\text { environment }\end{array}$ & Adaptive network operation \\
\hline Data redundancy & Data fusion and localized processing \\
\hline Security & Secure design \\
\hline $\begin{array}{c}\text { Integration with other networks and } \\
\text { Internet }\end{array}$ & $\begin{array}{c}\text { Efficient protocols and scalable } \\
\text { architectures }\end{array}$ \\
\hline Ad hoc architecture and large size & Small and low-cost sensor nodes \\
\hline $\begin{array}{c}\text { Quality of service requirement } \\
\text { Application-specific design }\end{array}$ \\
\hline
\end{tabular}

\subsection{Energy management in WSNs}

Nowadays application of the wireless sensor networks in the real life is quickly decreasing. However, the problem with energy source for WSNs and their sensor nodes is still as a barrier, which prevents the complete utilization of this new technology.

\subsubsection{Energy management in data acquisition and transmission}

Batteries are the typical sources to supply required energy of the sensor nodes. Although they use additional energy, which is harvested from the external environment (e.g. solar cells or piezoelectric mechanisms), there is still limited 
resource to be consumed wisely. Thus, the efficient energy management is the key factor of designing wireless sensor network.

Major number of the researches in this field, proposed energy management strategies when it is assumed that data transmission consumes significantly more energy than data acquisition. However, this assumption may not be true in many practical cases of the wireless sensor network's applications, as the consumed power of the sensing activities can be comparable or greater than that of radio [2]. Therefore, energy management in sensor level needs to consider to the amount of energy, which is consumed in all of the stages acquisition, process and transmission of data [7].

\subsubsection{Major sources of energy waste in WSNs}

Energy consumption in a sensor node can be either from "useful" or "wasteful" sources. Useful energy consumption may be resulted from transmitting or receiving data, data processing, processing query requests, and forwarding queries [25].

Wasteful energy consumption might be due to the reasons such as idle listening, collision (receiving more than one packet at the same by one node), overhearing (a node receives packets which is sent to other nodes) and some other wasteful sources. Considering to the aforementioned facts, an energy efficient design for the WSNs and deploying suitable routing protocol reduce the consumption of energy in both of the useful and wasteful sources. 


\section{Chapter 3}

\section{ROUTING PROTOCOLS IN WIRELESS SENSOR}

\section{NETWORKS}

Routing layer in the wireless sensor networks is a layer, which is responsible for transferring collected data from each sensor node to the base station. As packet of data can be transmitted through the different paths, making decision for selecting the path, may has an important effect on the load balancing between nodes and delay. Then the energy efficiency and supporting the mobility depends on the routing protocol and maintenance method, which is chosen for the system [8].

\subsection{Requirements}

Routing in a wireless sensor networks is basically different from the other wireless networks, as there are many specific requirements for wireless sensor networks. Main requirements of the WSNs are as follows.

\subsubsection{Data-centric communication}

Traditional wireless networks are node-centric and use this way for communication. It means unique address is used for each node, when they are sending and receiving data to each other. Mainly routing is based on data-centric communication in the WSNs. In this type of communication, sensed data are important whereas knowing the specific address is not necessary. 


\subsubsection{Resource constrained}

As the wireless sensor network has many restrictions with the resources, routing cannot be too much heavy operation in computation. In addition, it cannot store large information of the routing-state.

\subsubsection{Network lifetime}

In many applications of WSNs long term deployment is required which emphasizes the importance of network lifetime. Since all sending and receiving in a transmission consumes energy, the messaging overheads needs to be kept to a minimum by the routing protocol. It is known that routing protocol try to deliver data packet through the most energy-efficient path. Hence, several parameters must be considered to choosing the path. A link with low-reliability potentially causes to packet loss. Consequently, it causes consuming unnecessary energy, which will be used for retransmitting the packet. In the next coming chapters we will discuss that the energy consumption is proportional to the square of distance. So using an intermediate node for sending data reduces the energy consumption. Moreover minimizing the energy consumption only for a single node is not sufficient. The total energy of the system must be considered for measuring energy efficiency of the routing protocols. It is certainly important to prevent forwarding most traffic of data by a single or a few nodes. Otherwise, nodes with high traffic may die first or very soon, and the network will be separated into the different partitions. In this case, some of the remained nodes are not able to communicate with the sink node.

\subsubsection{Scalability}

Routing protocols are supposed to deal with the networks, composed of hundred or even thousands of nodes. Due to memory constraints and the several numbers of nodes, routing tables may not be used to maintain routing information. Then a 
distributed routing is needed and it is better to use the nodes only knowing their neighbors.

\subsubsection{Aggregation}

By the data-centric characteristic transmitted packets can be reduced by data aggregation. Size of packet in data aggregation maybe reduced by either any aggregation functions such as: maximum, minimum, sum or average, or by concatenation of many samples into one packet.

\subsubsection{Robustness}

WSNs most of the time work on the error-prone environments. Obstacles and changing the environmental condition are the common reason for occurrence of a transmission error. Moreover, one of the nodes may die regarding to low energy when it is not expected. Since in many cases sensor nodes are randomly deployed, sometimes interference is caused by densely populated areas, while in the area with less number of nodes, routing must use low quality links. Then routing protocol cannot trust on a single link and have to use redundancy in order to obtain a reliable routing.

\subsubsection{Mobility support}

A typical WSN consist of large number of immobile nodes. However, depending on the application mobile nodes or mobile sink may be required. Target tracking is the application in which a node is embedded to the monitored target, that is moving on every side of the network. Sink mobility may be needed when a mobile user moving around the network and collect the data from distributed nodes. 


\subsubsection{Application-specific behavior}

A WSN is specified by variety of many applications, and the routing protocols must be suitable for different types of applications. For instance monitoring in a static situation is very different with target tracking in dynamic environment.

\subsection{Classification}

Routing in WSNs can be classified according to their operation. Generally there are many routing algorithm for the wireless sensor networks. However, many of them could be categorized in seven important groups. In Table 3.1, these categories and some of the important protocols are presented [9]. A brief explanation about each category and some of the sample protocols from each category is selected according to its relevancy to the topic.

Table 3-1: Routing protocols for WSNs [9].

\begin{tabular}{|c|c|}
\hline Classification & Typical Protocols \\
\hline Location-based Protocols & GEAR, MECN, GAF \\
\hline Data-centric Protocols & EAD, Directed Diffusion \\
\hline Hierarchical Protocols & HEED,PEGASIS, LEACH, \\
\hline Mobility-based Protocols & Tree-Based Dynamic Proxy, TTDD, SEAD \\
\hline Multipath-based Protocols & Braided Multipath, Disjoint Multipath, \\
\hline Heterogeneity-based Protocols & CHR, IDSQ \\
\hline QoS-based protocols & Energy-aware routing, SAR \\
\hline
\end{tabular}

\subsubsection{Location based protocols}

By this method, each sensor node uses the mean of its location for addressing. Information of the location is necessary for the nodes in order to compute the 
distance between two particular nodes. So the consumption of energy can be roughly calculated. In this section some of the most important location based protocols is introduced.

\subsubsection{Geographic Adaptive Fidelity (GAF)}

GAF is a routing protocol at the beginning developed for mobile ad hoc network (MANET) that is an energy-aware protocol. But it can also be used for WSNs, regarding to this point that it has a preference for energy conservation. Geographic Adaptive Fidelity (GAF) is designed based on the model of energy that considers the energy conservation. This consideration is due to the transmitting and receiving of packets in addition to idle time while the radio sensor is on for finding if there are any incoming packets. Basically, GAF turn off the sensors which are not needed, when it keeps constant the routing fidelity level (uninterrupted connection of the sensors with transaction) [10]. In this protocol, sensor area is separated to grids and each of the sensor use location parameters that is obtained from GPS or other similar systems.

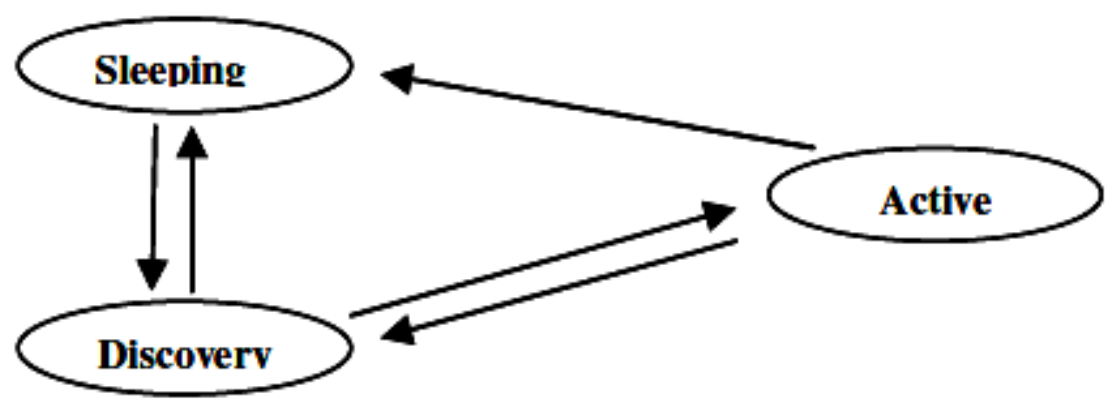

Figure 3.1. GAF-State transition diagram [10]

As illustrated in Figure 3.1 three states exist in state diagram of GAF. They are sleeping, discovery and active modes. When a sensor goes to the sleeping mode, its radio is turned off in order to saving energy. In the state of discovery, a discovery 
massage will be exchanged by the sensors, to learn about the other sensors. Also in the active state, sensors do same sending discovery massage regularly. Therefore GAF aims acceptable network lifetime, since only one sensor is active in each grid.

\subsubsection{Geographic and Energy-Aware Routing (GEAR)}

This method of routing is an energy-efficient protocol. In GEAR sensors need to have a localization instrument like GPS unit or other system [11]. Using this method also based on awareness of energy for each sensor as well as their neighbors for routing. Then recursive geographic forwarding algorithm is responsible for distributing the packet in the target region.

\subsubsection{Minimum Energy Communication Network (MECN)}

MECN is another location based protocol for randomly deployed WSNs. This is suitable for the mobile networks and tries to achieve the minimum energy for the network. It uses the best available spanning tree ended to the sink node named minimum power topology [12]. The topology considers the places of sensors in the field and contains two important states called enclosure graph construction and cost distribution.

Since MECN is a self-reconfiguring protocol, then depletion of the batteries is an important issue for the static networks. So sensed data is transmitted to the sink via the neighbor and after a while, the neighbor will die soon, and this part of network would be disconnected from the system. For this reason the enclosure graph as well as minimum power topology must be dynamic according to the remained energy for each sensor.

\subsubsection{Data-centric protocols}

These types of protocols are different from the address-centric protocols in traditional wireless networks. In the address-centric protocols each sensor that sense 
data is like a source node and independently is responsible for transmitting packet to the sink. While there are differences in data-centric protocols, and intermediate sensors do some aggregation on the data.

It means source sensors transmit their data to other intermediate sensor and after aggregating data from multiple source sensors, routing can be completed in to the sink. It can perform optimization in term of energy saving, since less transmission is needed. In the following, some of theimportant data-centric routing protocols are presented for the wireless sensor networks.

\subsubsection{Energy-Aware Data-Centric Routing (EAD)}

This routing protocol is a new method based on making a virtual backbone, mainly comprised of active sensors, which respond to the processing of data and relaying of traffic [13]. This protocol has three main definitions which are namely tree, backbone and leaf nodes. The network is defined by a tree, containing all of the nodes with the leaf nodes that their radio transmitter is turned off. Nevertheless, backbone is composed of active nodes with turned on radios. EAD with these infrastructures tries to construct an optimized tree achieved from the spanning tree with less number of leaf nodes, and thus minimum number of active nodes. Therefore size of the backbone is reduced by reducing the number of active nodes. The protocol is specifically energy aware and attempts to improve the network lifetime.

\subsubsection{Directed Diffusion}

Directed diffusion is data-centric routing protocol, which cope with principal requirements of WSNs such as scalability, robustness and energy efficiency. At the start point of process in directed diffusion, a low data rate is specified by the sink for 
incoming events. Therefore, the sink node is able to reinforce one specific sensor to transmit events with the higher rate of data.

Moreover, if a next sensor obtains this message of interest and realizes the interest of sender has higher rate of data than the previous one, and also this rate will be higher than of other available gradient, it would reinforce one of its own neighbors or even more [14].

\subsubsection{Hierarchical protocols}

Hierarchical clustering protocols are the topic of many researches in the last few years. It has been considered from different point of views.

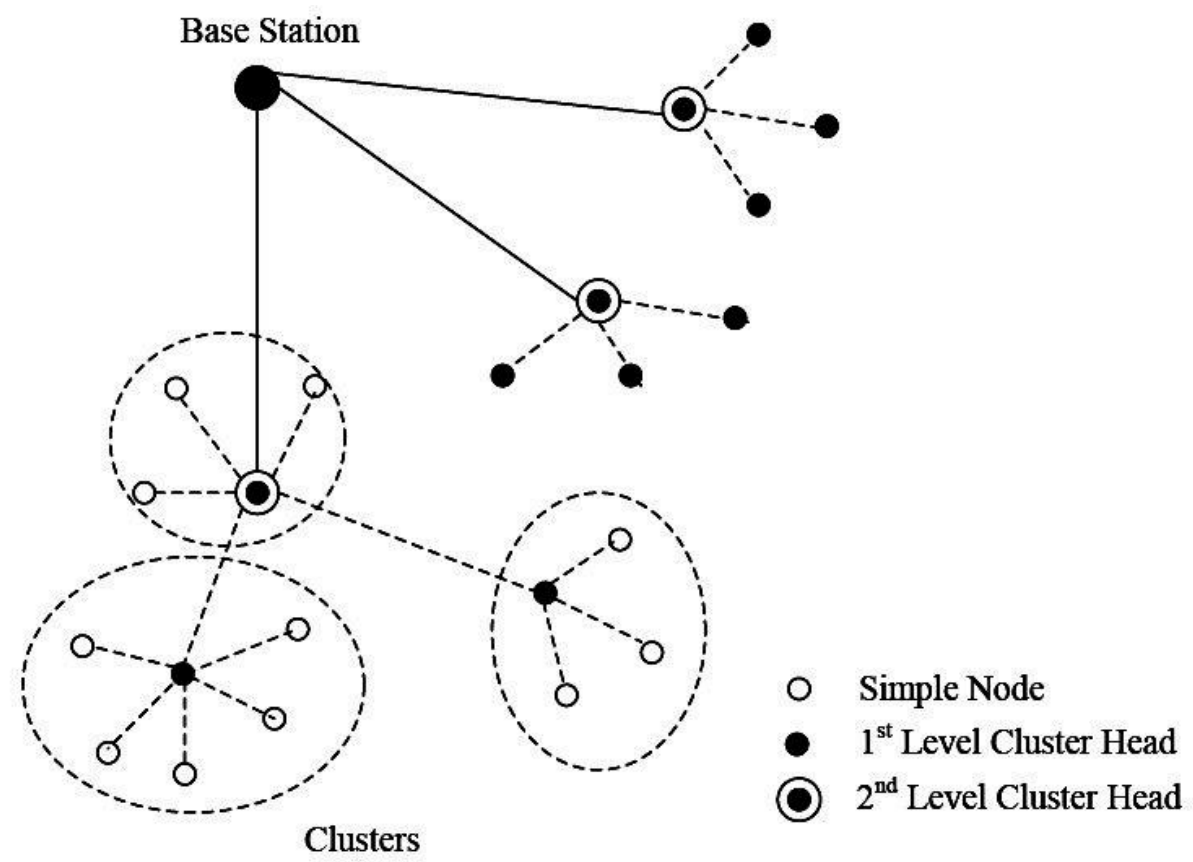

Figure 3.2. Cluster-based Hierarchical Model [1]

It is a method, in which sensors forward sensed data to the base station using energyefficient communication. In this part, some of well-known samples of this type of protocols will be reviewed. It will be described that in many of them, the networks are divided into the few or many clusters. For each cluster (clump), one specific node 
is defined as a head of cluster and this head will manage the cluster. Cluster head is in charge of managing all data transmission activities of their cluster nodes.

As shown in Figure 3.2 the idea of the hierarchical divides network into the clusters. Lower level cluster head is responsible to send data to the higher level cluster head. According to existing levels in the network higher level cluster heads will forward data to the base station. It is possible for data to be transmitted from node to node. But jumping from a cluster layer to the other one covers large distance and it causes fast movement through the network and finally to the base station. In the following samples of hierarchical-based routing protocols are presented [15].

\subsubsection{Low-energy adaptive clustering hierarchy (LEACH)}

The first and most desired hierarchical clustering protocol is Low-energy adaptive clustering hierarchy (LEACH) which was developed with approach to energyefficient algorithm. LEACH uses data aggregation (or fusion) and sends only useful data with smaller size among the sensor nodes. Based on the duration, clustering task is rotated between the nodes. Cluster heads use direct transmission for sending data to the sink node. However they use clusters to extend the network lifetime. As LEACH is hierarchical routing protocol it will split the network to number of sensor clusters, that are built up by using localized coordination [16].

LEACH is totally distributed and doesn't need any global knowledge of network. It decreases consumption of energy by two main operations (I) reducing the energy consumption between the cluster head and cluster nodes and (II) turning off the radio of the nodes which are not head in the current phase. It should be considered that LEACH is not applicable for the network that is distributed in the large area. Because 
it uses single-hop routing in which all nodes are able to send data directly to cluster heads and base station.

\subsubsection{Power-Efficient Gathering in Sensor Information Systems (PEGASIS)}

PEGASIS is a routing protocol that is extension of LEACH. In PEGASIS a chain of sensors is created that send and receive data from their neighbor [17]. But only one node from the chain is responsible to forward data to the base station. In this protocol data is collected, moves between nodes, aggregated and finally is sent to the sink. Against of LEACH, PEGASIS employ one node form the chain to send data to sink instead of using variable nodes and it prevents from constructing a cluster. In this method data is sent to its neighbors in data aggregation and not to be sent to $\mathrm{CH}$ as in LEACH. Furthermore in this routing protocol every node has global knowledge about the other nodes in the network especially about the location of sensors. When battery depletion happens to a node and it fails to operate, the chain is formed utilizing the greedy approach with bypassing died sensor. In each round one sensor which is randomly selected will send the aggregated data to the sink, hence reduction of energy consumption per each round is comparable to LEACH. Investigation on the results declaring that PEGASIS can provides two times network lifetime as much the same network lifetime deploying LEACH protocol. Such result can be caused by removal of the parameters of the dynamic clustering in LEACH and minimizing the number of transmitting and receiving aggregated data. However clustering overhead is not necessary, PEGASIS needs dynamic adjustment because each of the nodes must know about energy level of its neighbor for finding the rout.

\subsubsection{Hybrid, Energy-Efficient Distributed Clustering (HEED)}

This routing protocol is based on LEACH principals, with using residual energy and degree of the nodes for selecting a cluster, in order to obtain power balancing. HEED 
[18] is developed based on four important parameters: (a) extending lifetime of the network by dispreading consumption of energy, (b) terminating the process of clustering while number of iterations is constant, (c) keep to minimum the control overhead, (d) constructing cluster heads which is well distributed and compact cluster.

In HEED two parameters are important for choosing $\mathrm{CH}$ that is changing periodically. The first one is the residual energy of the sensors, and the second parameter is cost of communication in the cluster. In the primary parameter probabilistic selection is used to initialize the cluster heads, but second one is deployed for breaking ties. HEED has better performance of network lifetime in comparison to LEACH clustering, since LEACH uses random selection of $\mathrm{CHs}$ and it may causes sooner death for some of the nodes.

\subsubsection{Mobility-based Protocols}

In design of wireless sensor networks, mobility always leads to new challenges for routing protocols. When mobility is required for the sink, energy efficient protocols which can secure delivery of the data to the mobile sink are also needed. In this section some of mobility-based protocols in wireless sensor networks are given.

\subsubsection{Scalable Energy-Efficient Asynchronous Dissemination (SEAD)}

Scalable Energy-Efficient Asynchronous Dissemination is mobility-based routing protocol that is self-organizing and is developed to trade-off between energy saving and making minimum the forwarding delay to the mobile base station [19].

\subsubsection{Dynamic Proxy Tree-Based Data Dissemination}

One of the mobility based routing protocol is dynamic proxy tree-based data dissemination [20] method which is used for maintaining a tree consist of a sensor source connected to numerous sinks. It lets the source to send its data freely to 
mobile sinks that are interested in the sensed data from a source. It is showing that network consists of many mobile sinks along with the stationary sensor nodes. Role of the sensors are detecting data and monitoring mobile target without interruption, while the sinks with mobility are responsible to gather data from particular sensors.

In this framework as the targets are moving, the source may be changed to the new sensor which is closer to the target and the closer one will become the source. All of the sources are represented by stationary source proxy and every sink with stationary sink proxy. A new source proxy is applied to a source just when the interval of the source and its current proxy will be greater than a certain threshold. Similarly for the sink proxy will be applied when the distance become more than specific threshold.

\subsubsection{Multipath-based protocols}

In view of communication between sensor nodes and base station, two method for routing is considered which namely single-path and multi-path routing. It can be seen from the name that single-path routing uses shortest path in order to transmit data from source sensor to the sink. However multi-path routing detects the prime $\mathrm{k}$ shortest path from source node to the sink and splits up the load between the paths smoothly. In this part some sample of multipath routings in WSNs are reviewed.

\subsubsection{Disjoint Paths}

Disjoint path is a multipath routing protocol in which routing protocol tries to find minimum number of path as the alternates with no common sensor with together and also with the first path. The best available path form latency and efficiency point of view is considered as a primary path, while the less efficient and desirable paths are deemed as alternates. The alternate paths are made independent from the prime path by the disjoint protocol. Hence for any reason when a failure happens in the first 
path, it doesn't have any effect on the alternate paths. Thus disjoint paths can be really flexible to sensor failure.

\subsubsection{Braided Paths}

To establish the braided multipath, at the beginning the prime path is determined. So, for all nodes in the primary pathway, the most desired path from source node to the BS that is not contained that node will be figured. These best optional paths are not certainly separated from the first pathway and they are known as idealized braided multipath. Furthermore the links for every alternate paths lie on or either in the clos location to the primary path.

\subsubsection{Heterogeneity-based protocols}

There are two different types of sensors in the wireless sensor network with heterogeneity network architecture [9]. In the first type which is called line-powered sensors there is no any energy constraint. However in the second type named batterypowered sensors they have limitation in lifetime, and regarding to this matter they need to use their own energy as efficient as possible. This group of sensors tries to minimize their potential of data computation and communication. In this part one of Heterogeneity-based protocol is reviewed and how it can extend the network lifetime will be discussed.

\subsubsection{Cluster-Head Relay Routing (CHR)}

There are two sorts of sensors in cluster-head relay routing protocol which construct a heterogeneous sensor network with a single sink. The first category indicated by Lsensors and consists of many low-end sensors. Second type is denoted by H-sensors with few number sensors. All sensors categorized in two groups are static and also they are aware about their locations by deploying location service. Furthermore both of H-sensors and L-sensors are randomly and uniformly distributed within a network 
area. In the WSN using CHR protocol, heterogeneous is divided into clusters which is formed of $\mathrm{H}$-sensor as a head along with number L-sensors. In a cluster L-sensors are responsible to transmit sensed data by one of L-sensor to the $\mathrm{H}$-sensor which is acting as a cluster head. In addition the responsibility of $\mathrm{H}$-sensor is to data fusion within its own cluster and to forward aggregated data to the sink originating from other cluster heads. In CHR protocol L-sensors transmit data via short-range to their h-sensor neighbors, however H-sensors use long-rang data transmission to the other H-sensor neighbors and to the sink [22].

\subsubsection{QoS-based protocols}

In a routing protocol for WSNs beside the energy efficiency it is also significant to think about the quality of service (QoS) requirements in terms of reliability fault tolerance and delay. Quality of service (QoS)-based protocols try to make a balance between the QoS and energy consumption.

\subsubsection{Sequential Assignment Routing (SAR)}

Sequential Assignment Routing (SAR) is known as of the primary routing protocol of WSNs that represent QoS requirements. This routing protocol is a multi-path approach which is trying to obtain fault tolerance and energy efficiency. In SAR choosing a rout depends on three parameters namely QoS on each path, energy resources and priority level of each packet data [23].

\subsubsection{Energy-Aware QoS Routing Protocol}

In this routing method, real-time traffic is produced whit imaging sensors [23]. Energy-Aware QoS Routing Protocol detects the best path in terms of cost and energy efficiency which meets exact end to end delay within connection. Hereby, cost includes the nodes' energy reserve, error rate, energy of transmission and other parameters needed for communication. A class based queuing model is deployed in 
order to meet simultaneously both real time traffic and best effort. The queuing model provides permission to sharing of service for both non real-time and also real time traffic. This method, discover a record of pathways with minimum cost and take one of the path from the obtained list that meets the requirements of end-to-end delay. 


\section{Chapter 4}

\section{ENERGY ANALYSIS OF THE DIRECT AND MINIMUM TRANSMISSION ENERGY ROUTING \\ PROTOCOLS}

There are large numbers of researches in which design principals, and technical approaches of routing protocols of WSNs have been discussed. In chapter three, some of the popular protocols were classified and relevant examples were given. In this chapter, it is important to take into consideration that most of the researches, analysis and simulations are based on randomly distributed wireless sensors networks. However, in some applications, sensor nodes can be evenly distributed through the field. In this research, some of basic equations and facts are obtained from a linear evenly distributed sensor network, which is in one-dimensional space. After that, experiments and proposed method will be extended to the randomly distributed network within two-dimensional space. Moreover, some other parameters such as massage length, number of nodes, and frequency of transmission are manipulated and finally energy efficient routing protocol will be presented.

\subsection{Energy calculation}

In a wireless channel, electromagnetic wave's power decrease in accordance with the power law function of the distance between the receiver and transmitter. The function specifies the energy dissipation while a signal is transferring between transmitter and receiver in direct line or multipath model. Energy consumption in 
both models obeys the power law function. The definition of power law function is depended on a critical distance between receiver and transmitter [26], which is named $d_{\text {crossover }}$, and defined as:

$$
d_{\text {crossover }}=\frac{4 \pi \sqrt{L h_{r} h_{t}}}{\lambda}
$$

In this equation, $L$ is the system loss factor in the propagation model, $h_{t}$ is the height of transmitter and $h_{r}$ is the height of the receiver antenna over the ground. $\lambda$ Is the signal wavelength, which is defined by light speed (c) and frequency of transmission $\left(f_{t}\right)$ as follows:

$$
\lambda=c / f_{t}
$$

In the following table path loss exponents for some of useful environments are given:

Table 4-1: Different environments and corresponding path loss exponent. [26]

\begin{tabular}{|c|c|}
\hline Environment & Path loss exponent \\
\hline In building line-of-sight & 1.6 to 1.8 \\
\hline Urban area cellular radio & 2.7 to 3.5 \\
\hline Free space & 2 \\
\hline Shadowed urban cellular radio & 3 to 5 \\
\hline Obstructed in factories & 2 to 3 \\
\hline Obstructed in building & 4 to 6 \\
\hline
\end{tabular}

There are two different models which can be used for energy attenuation according to the calculated crossover distance. These models are namely Friis free space model and two-ray ground propagation model. When the distance between receiver and transmitter is less than crossover value the first model is considered and attenuation of energy level is corresponding to $d^{2}$. However as the transmission distance becomes greater than $d_{\text {crossover }}$ the second (two-ray ground propagation) model will be used and $d^{4}$ is proportional to the energy attenuation [16]. 
In a standard type of a wireless sensor node such as TelosB operation frequency is varying in the interval 2400 to $2483.5 \mathrm{MHz}$. For the simplicity of the calculation it is assumed that the height of the receiver and transmitter antenna is 1 meter above the ground. In addition, system is considered as to be lossless which means $\mathrm{L}=1$. Therefore using equation 4.1 the value of crossover distance is calculated to be $d_{\text {crossover }}=104.6 \mathrm{~m}$, while in this research all of the distances are taken less than this value. In addition for an industrial application such as shop floor, distances between the machines that the wireless sensor nodes might be embedded on them would be less than the above distance. As a result in this research $d^{2}$ attenuation is used for the all future calculations and simulations.

In Table 4-2 different values of crossover distances for some of sensor nodes working on variable frequencies are given. Then using the transmission frequency and calculated crossover value distance, amount of path loss can be specified.

Table 4-2: $d_{\text {crossover }}$ for different frequencies [24]

\begin{tabular}{|c|c|}
\hline Carrier Frequency & d_crossover \\
\hline $900 \mathrm{MHz}$ & $37 \mathrm{~m}$ \\
\hline $1300 \mathrm{MHz}$ & $54 \mathrm{~m}$ \\
\hline $1700 \mathrm{MHz}$ & $71 \mathrm{~m}$ \\
\hline $2100 \mathrm{MHz}$ & $87 \mathrm{~m}$ \\
\hline $2500 \mathrm{MHz}$ & $104 \mathrm{~m}$ \\
\hline
\end{tabular}

Figure 4.1 shows the basic radio energy model, which is used in this research. As it is illustrated in this figure, $d$ is the distance between transmitter and receiver antennas. Considering to the transmitter part of a sensor node there are two elements which is consuming energy. Electric circuit consumes energy in order to process one bit data and is determined by $E_{\text {elec }}$. Therefor processing k-bit packet consumes $E_{\text {elec }} * k$ 
amount of energy. In addition amplifying and transmitting k-bit packet over distance $d$ consumes energy that is defined by $\epsilon_{a m p} * k * d^{n}$.

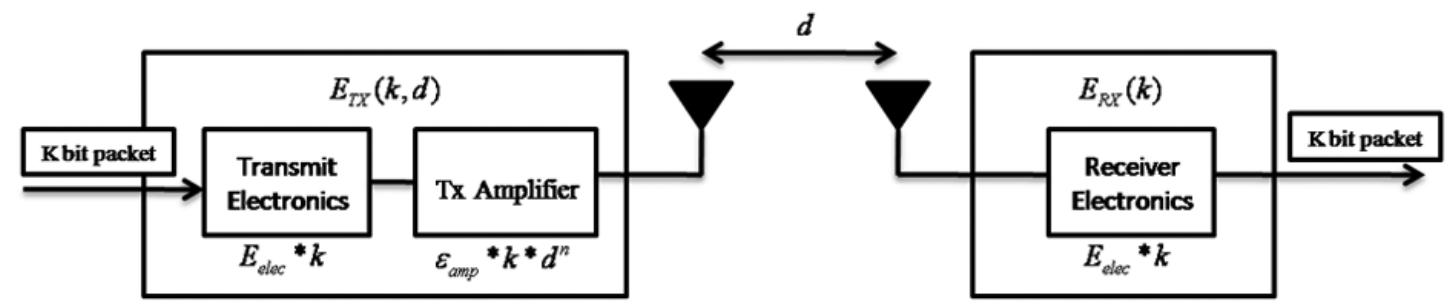

Figure 4.1. Radio Energy model [16]

As it was mentioned before in this study distance between transmitter and receiver is considered to be less than $d_{\text {crossover }}$ and so Friis free space model and $d^{2}$ attenuation will be used. Therefore the energy which is used in transmitter part in order to transmit k-bit packet can be calculated from the equation:

$$
E_{T X}(k, d)=E_{\text {elec }} * k+\epsilon_{a m p} * k * d^{2}
$$

Likewise in the receiver part energy dissipation in order to process k-bit packet which is received from the transmitter can be defined by the equation:

$$
E_{R X}=E_{\text {elec }} * k
$$

By adding these two equations $E_{T X}$ and $E_{R X}$ total amount of energy consumption over transmission distance $d$ can be calculated.

\subsection{Analysis of routing protocols in WSNs}

Design of routing protocols is a key factor of energy efficiency in wireless sensor networks. Due to the fact, that there is energy limitation for sensor nodes, it is a crucial challenge to optimize routing protocols in energy consumption. Hence by conserving energy for each node the lifetime of network would be decreased. It should be mentioned that for different environments, different routing methods are designed and selection of the method, certainly depends on the application. 


\subsubsection{Direct transmission}

In this thesis, two popular routing protocols, namely direct transmission and minimum energy transmission (MTE) are selected and will be utilized in this research. These two methods are desirable for many applications. Figure 4.2 shows a single direct transmission in the linear network which is evenly distributed.

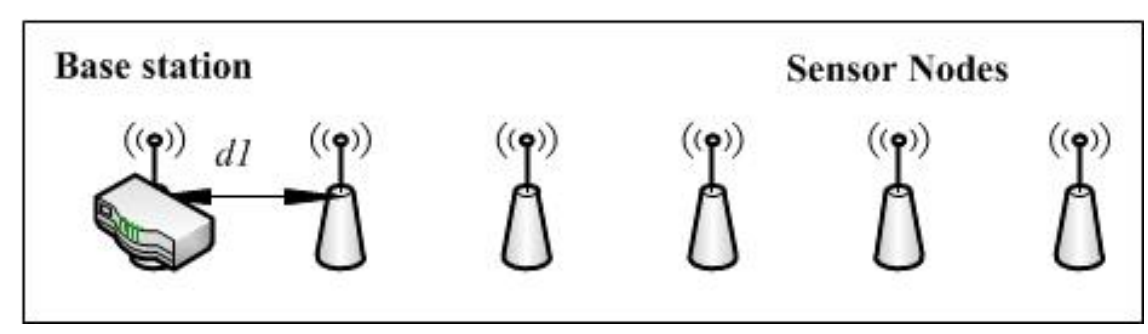

Figure 4.2 Direct transmission method in a linear WSN [16].

As it is shown in Figure 4.3 in direct transmission each nodes send its own data directly to the sink node that is responsible to collect all data and forwarding them to the PC for more processing. It is clear that each node consumes amount of energy for transmitting data to the sink node. The amount of energy consumed by each node is corresponding to the distance of the node with the base station. It can be resulted from equation 4-3 that total amount of consumed energy will become more, as the nodes are distributed in the long distance from the base station. Therefore, this method is not efficient for vast environment such as forests, lakes, etc. Moreover, long distance may cause to fail transferring data, when a sensor and base station goes far from each other more than coverage area of a sensor node. 


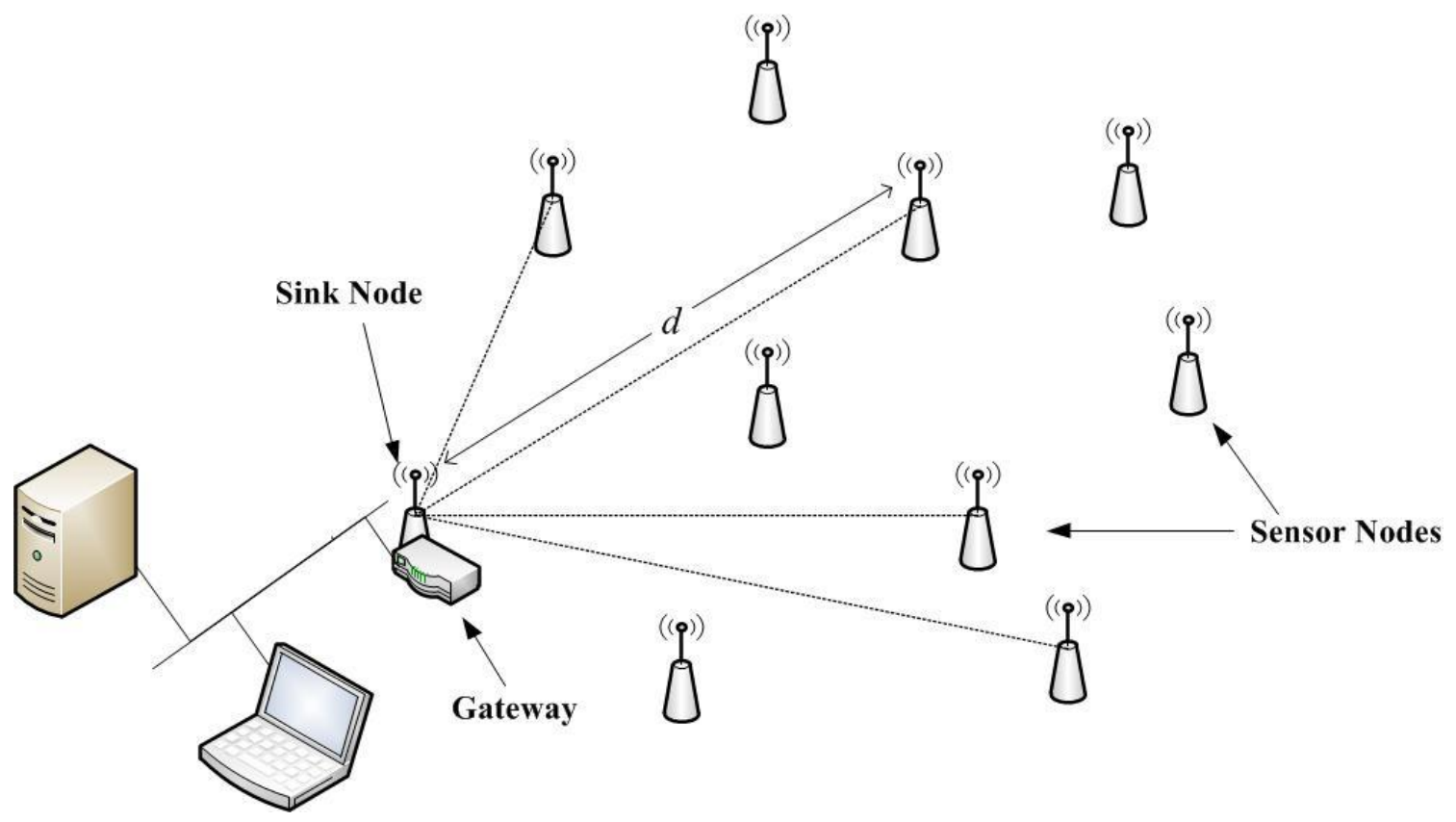

Figure 4.3. Direct transmission method in a distributed WSN

From the Figure 4.3 and equation 4.2, it is clear that the amount of energy consumed in a direct transmission in order to send k-bit packet of data is consumed in the electronic circuit and transmitter amplifier, which is equal to:

$$
E_{\text {direct }}=k *\left(E_{\text {elec }}+\epsilon_{\text {amp }} * d^{2}\right)
$$

Where $\mathrm{k}$ is the number bits in a packet, and is proportional to $E_{\text {direct }}$.In the network analysis, the parameter that is often considered is the total energy consumption of the system. Therefore, by rewriting equation (4.5) that is only for a single node data transmission over distance $d$, the following equation for the energy consumption of the system consisting $n$ number of nodes will be achieved [24]:

$$
E_{n \text { to B (direct })}=k *\left[E_{\text {elec }}+\epsilon_{\text {amp }} *\left(\sum_{i=1}^{n} d_{i}\right)^{2}\right]
$$

\subsubsection{Minimum transmission energy method (MTE)}

Minimum transmission energy is a method in which when one of the nodes is in the position to transmit data to the base station, it is called source node. In MTE, source 
node has to find the shortest path to the base station using some intermediate sensor nodes. Figure 4.4 shows that how a source node in the basic linear wireless sensor network, transfers its data to the base station through the other sensor nodes.

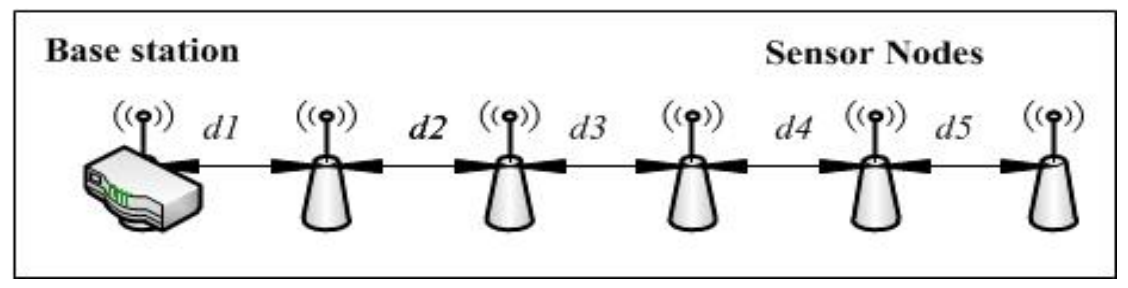

Figure 4.4. Direct minimum transmission energy (MTE) in a linear WSN [24].

In this method instead of using high energy path including one transmitter and one receiver, the massages of each node is transferred through the several intermediate nodes with low energy consumption via the shortest path. For example, if in the Figure 4.4 the last node, which is the $5^{\text {th }}$ node, tries to transmit its data, it will use 4 other intermediate nodes with different distances as a path to the base station. Therefor considering k-bit transmission from node $n$ to the base station, makes use of $n-1$ other nodes and the relevant consumed energy through the path is obtained from equation (4.7)

$$
E_{n \text { to } B(M T E)}=E_{n \text { to } n-1}+\cdots+E_{2 \text { to } 1}+E_{1 \text { to } B}
$$

Clearly $E_{n \text { to } B(M T E)}$ is the required energy in order to send k-bit data from node $n$ to the base station. Obviously using such a path take $n$ transmits and $n-1$ receives. Substituting equation (4.3) and (4.4) into equation (4.7) returns [16]:

$$
\begin{gathered}
E_{n \text { to } B(M T E)}=\left[k\left(E_{\text {elec }}+\epsilon_{\text {amp }} d_{n}^{2}\right)\right]+\cdots+\left[k\left(E_{\text {elec }}+\epsilon_{\text {amp }} d_{2}^{2}+E_{\text {elec }}\right)\right]+ \\
{\left[k\left(E_{\text {elec }}+\epsilon_{\text {amp }} d_{1}^{2}+E_{\text {elec }}\right)\right]}
\end{gathered}
$$

In the above equation, all of the intermediate nodes spend energy for transmitting and receiving data, except the first node that only consumes transmission energy. Therefore after simplifying of equation (4.8), $E_{n \text { to B(MTE) }}$ will be [24]: 


$$
E_{n \text { to } B(M T E)}=k *\left[(2 n-1) E_{\text {elec }}+\epsilon_{\text {amp }} \sum_{i=1}^{n} d_{i}^{2}\right]
$$

Now considering to the randomly distributed wireless sensor network through a 2D space, more attention for applying the formula (4.9) to the network is required. Figure 4.5 shows a randomly distributed WSN and illustrates how a path is formed by using MTE method.

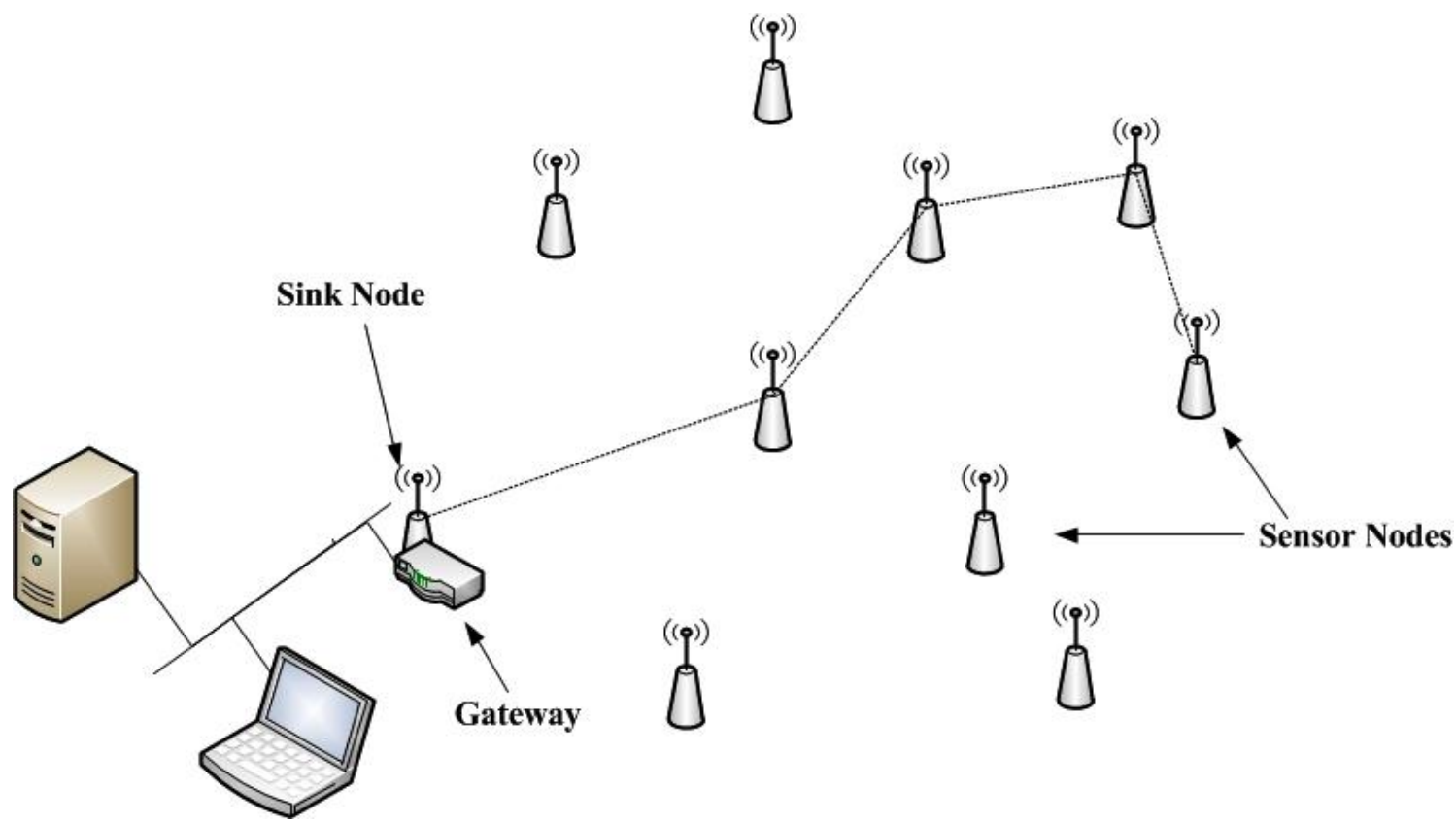

Figure 4.5. Randomly distributed WSN using MTE method for data transmission

As it shown in the picture when a source node desires to transmit its data to the base station, it will use some other intermediate sensor in order to forming its own path. For this purpose each node participating in the route, needs to find the closest node and send its data to this neighbor. Nevertheless, the discrepancy appears, when one node finds the nearest node, and it is not suitable for continuing the path. Because, however this node is the nearest one to the node which is transmitting its data, but it is not close to the base station. It means, the distance of this closest neighbor to the 
BS, is more than sender node to BS distance, and by choosing this node, the path will be longer.

Figure 4.6 clarifies the existing of this problem in design of these kinds of routing protocols. For example in this figure after passing $d 1$ and $d 2$ distances, node number 3 is supposed to find $d 3$. Although $d 3^{\prime}$ is the distance of the nearest node to $3^{\text {rd }}$ node, but it is not the best choice, since it is not leading the path to the base station within the most efficient way. Therefor as it is shown in the figure, $d 3^{\prime}$ will be ignored and $d 3$ will be chosen for continuing the path into the BS.

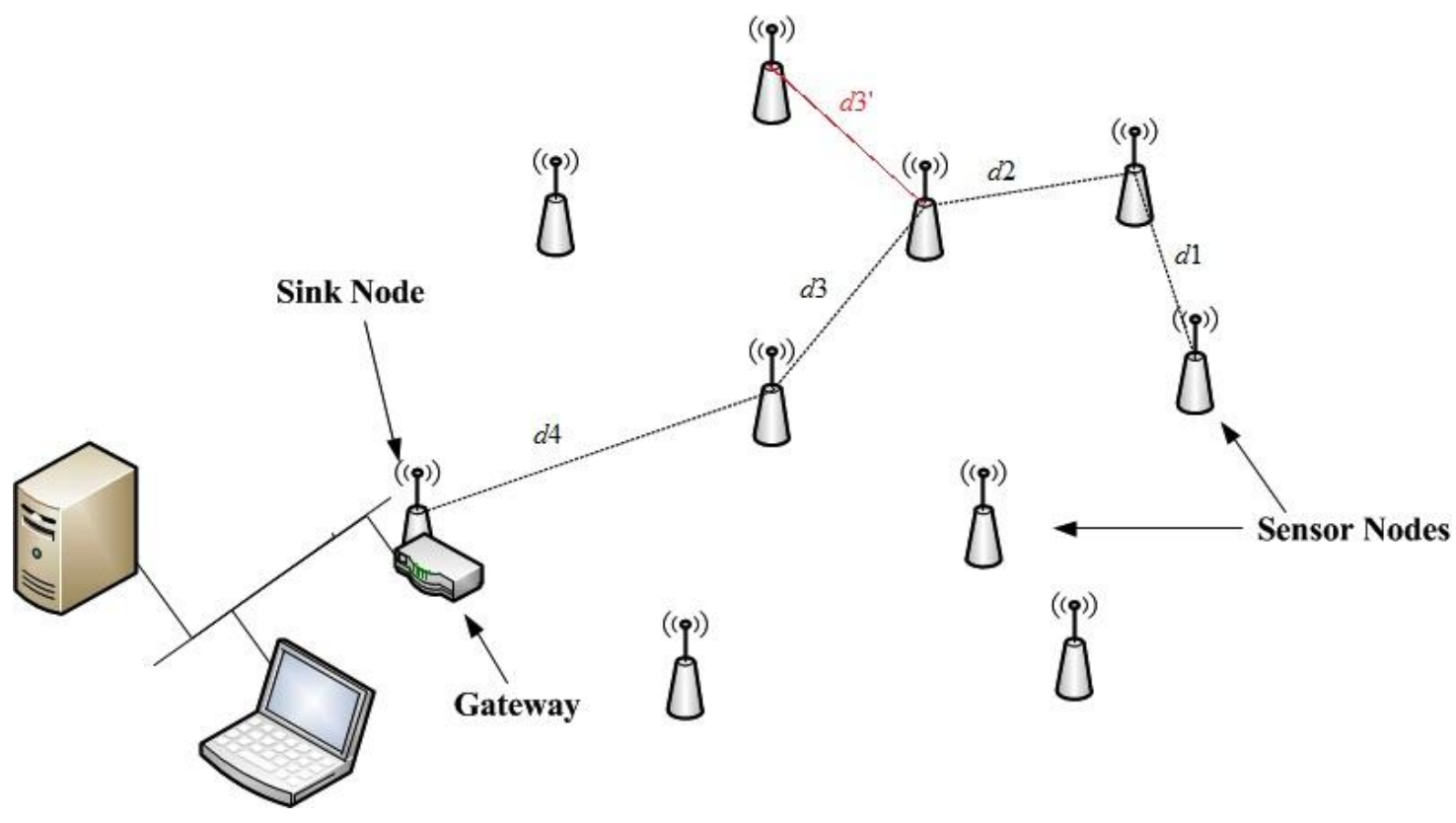

Figure 4.6. Finding path in the MTE routing protocol

Designing MTE protocol for the randomly distributed WSN in 2D space depends on the network size and number of nodes, and it has its own challenges and criteria. For this purpose, an algorithm is proposed for finding the most desired path for a random start point or source node. 
Figure 4.7 shows this algorithm of the MTE method as it is used in the design of new protocol in this research.

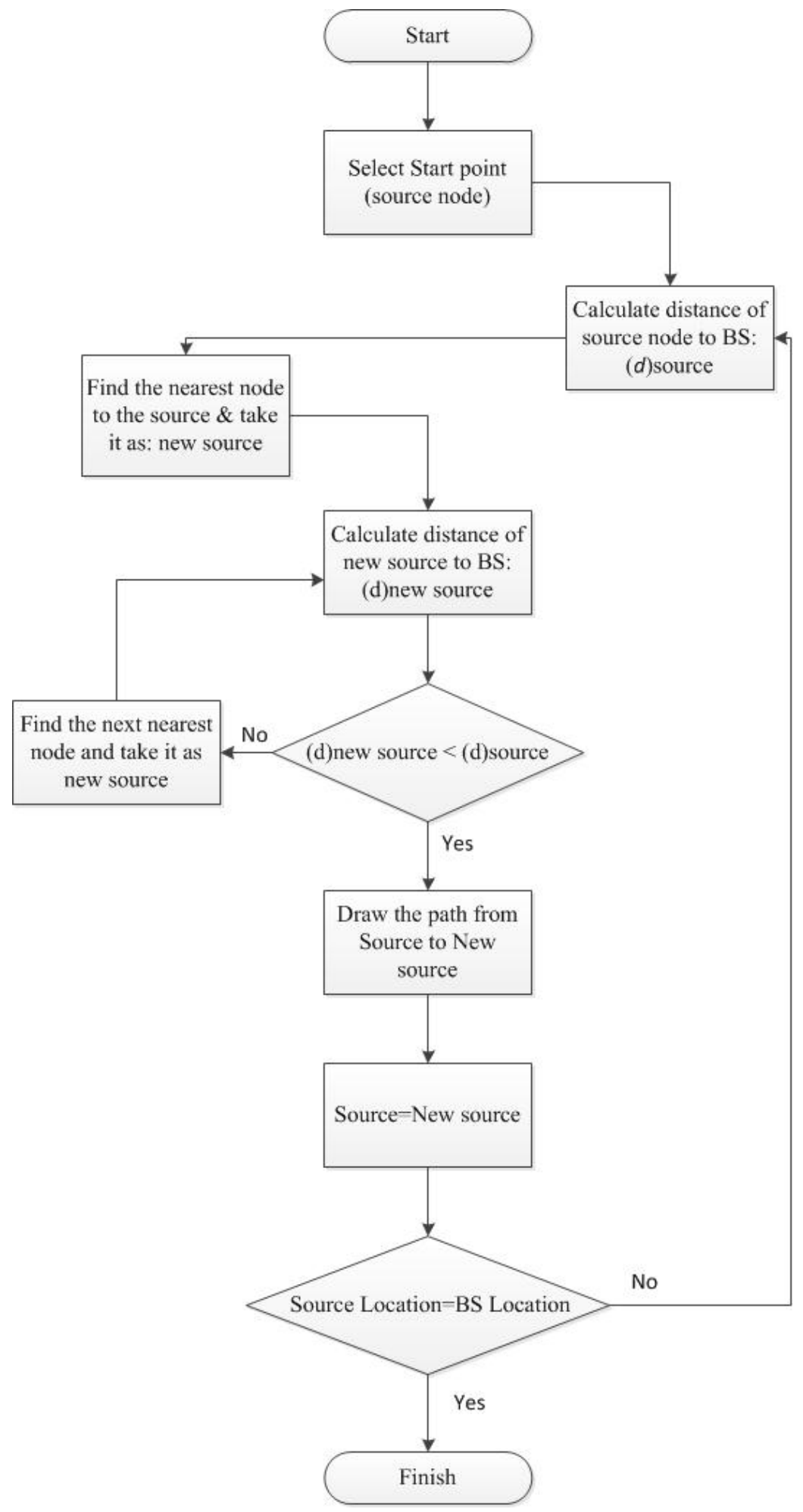

Figure 4.7. Algorithm of path finding in MTE method 


\subsubsection{Simulation of Direct and MTE routing protocols}

For simulation of this algorithm, Matlab software is used, and the obtained results from this simulation are very beneficial. An important point which must be considered in this part, is the replacing $n$ in the equation (4.9). For this purpose number of nodes, which compose the routing path, should be considered and take place of $n$, in the formula. This number is certainly different from the total number of nodes in the network. However, total number of nodes is one of main characteristics of the network which will be used in the network analysis.

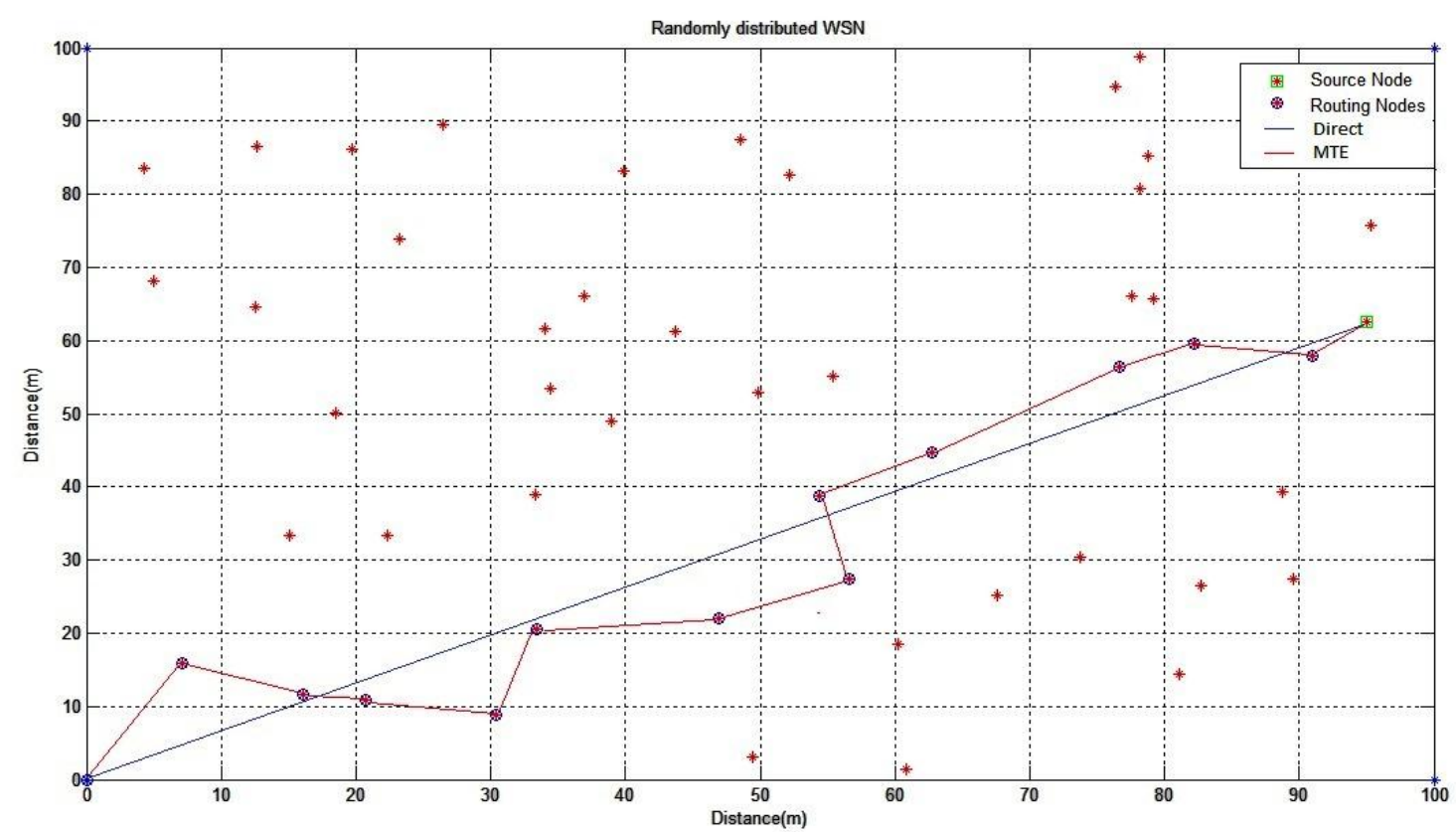

Figure 4.8. Simulation of direct protocol and path finding algorithm in MTE method When one random single node considered as to be source node (start point), calculating the energy consumption for each of direct and MTE methods results important facts, which are as follows.

a) As randomly chosen source node selected far from the base station, number of needed intermediate sensor nodes for routing to BS is increased. Therefore $E_{n \text { to } B(M T E)}$ and $E_{n \text { to } B(\text { direct })}$ will be computed and results shows that, as the number of nodes goes up, MTE method becomes more efficient in comparison to direct method. 
b) In the Figure 4.9 sensor nodes are distributed within $\left(100 \mathrm{~m}^{2}\right)$ network area, and for example 13 sensor nodes form the path to the BS. Expanding length and width of the network area, while keeping constant the other parameters, demonstrates that MTE is more efficient for the larger area.

c) Direct transmission protocol and the corresponding consumed energy in this method $E_{n \text { to } B(\text { direct })}$ is proportional to $\left(\sum_{i=1}^{n} d_{i}\right)^{2}$, while energy dissipation in MTE method is proportional to $\sum_{i=1}^{n} d_{i}^{2}$. It should be considered that $d_{\text {average }}$ in MTE method can be achieved from equation:

$$
d_{\text {average }(M T E)}=\left(\sum_{i=1}^{n} d_{i}\right) / n
$$

Where $n$ is the number of nodes get involved in the routing path construction. Considering to the average distance is certainly helpful in making decision of using direct or MTE method in different applications.

\subsection{Energy analysis of the System}

In some applications deploying a routing protocol for transmitting data from specific source node, might be appropriate, however this routing protocol is not generally efficient for the whole system. As it was mentioned before, one of the main characteristic of the wireless sensor network, which make it energy efficient or not, is the energy dissipation of the network. Therefore, in this chapter general information, which is obtained from single source node analysis, will be used to evaluate energy efficiency of all sensor nodes consisting in the network. Adding all results collected from performance of each sensor node, network compatibility with the applied routing protocol can be evaluated. Therefore, routing protocols can be compared together, while massage length or network size is changing. In this thesis for all simulations, transmitter/receiver electronic energy is assumed to be $E_{\text {elec }}=$ $50 \mathrm{~nJ} / \mathrm{bit}$, and amplifier energy consumption is taken to be $\epsilon_{a m p}=100 \mathrm{pJ} / \mathrm{bit} / \mathrm{m}^{2}$ (Same as References [16],[24]). Network size is the area in which sensor nodes are 
distributed and depending on the simulation purpose, can be fixed or variable between $1 m \times 1 m$ to $160 m \times 160 m$ sizes. Length of message or number of bits (k) is taken to be $\mathrm{k}=100$ bit and simulation will be carried out with 50 randomly distributed sensor nodes through the network area. In this section, applying pathfinder algorithm (Figure 4.7) to all sensor nodes, means that each node once act as source node. Then amount of energy that each node needs to transmit 100 bit massage to the base station will be calculated for both direct and MTE method. Therefore, by adding the energy of each node, total consumed energy of the system can be calculated for these protocols.

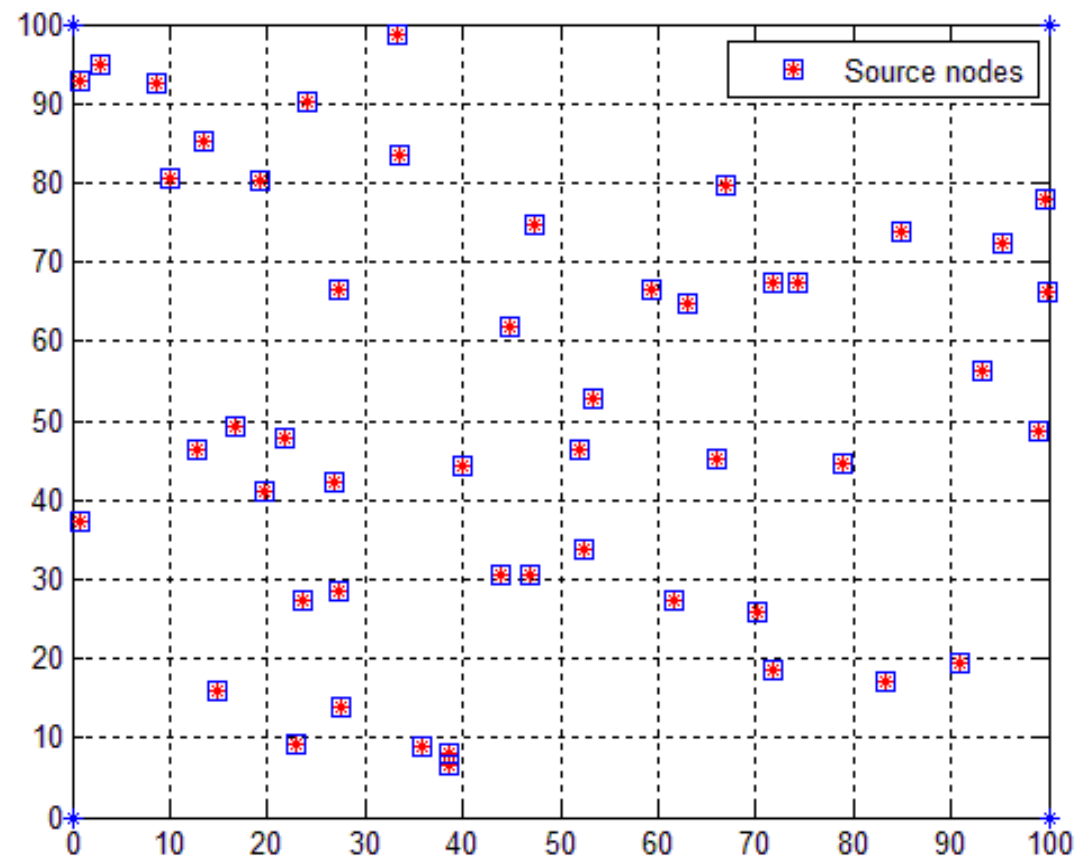

Figure 4.9. All of the nodes acting as source node one by one

Figure 4.9 shows that all nodes are taken to be source one by one, and the energy graphs will be drawn.

Considering to the facts, which was resulted from one single node simulation, it is now important to see, how is the efficiency of direct and MTE methods for the 
system, when the network size is changing. For this purpose three critical network sizes are chosen and the results are illustrated in the next coming figures.

For the first experience network size is selected to be $50 \times 50 \mathrm{~m}^{2}$ and 50 sensor nodes distributed over the field. In this case, average distance of the nodes from each other estimated to $4 m$, and then performance of energy efficiency of each protocol is evaluated. Figure 4.10 shows the amount of energy consumed for each node, which is sending its data using both of direct and MTE method.
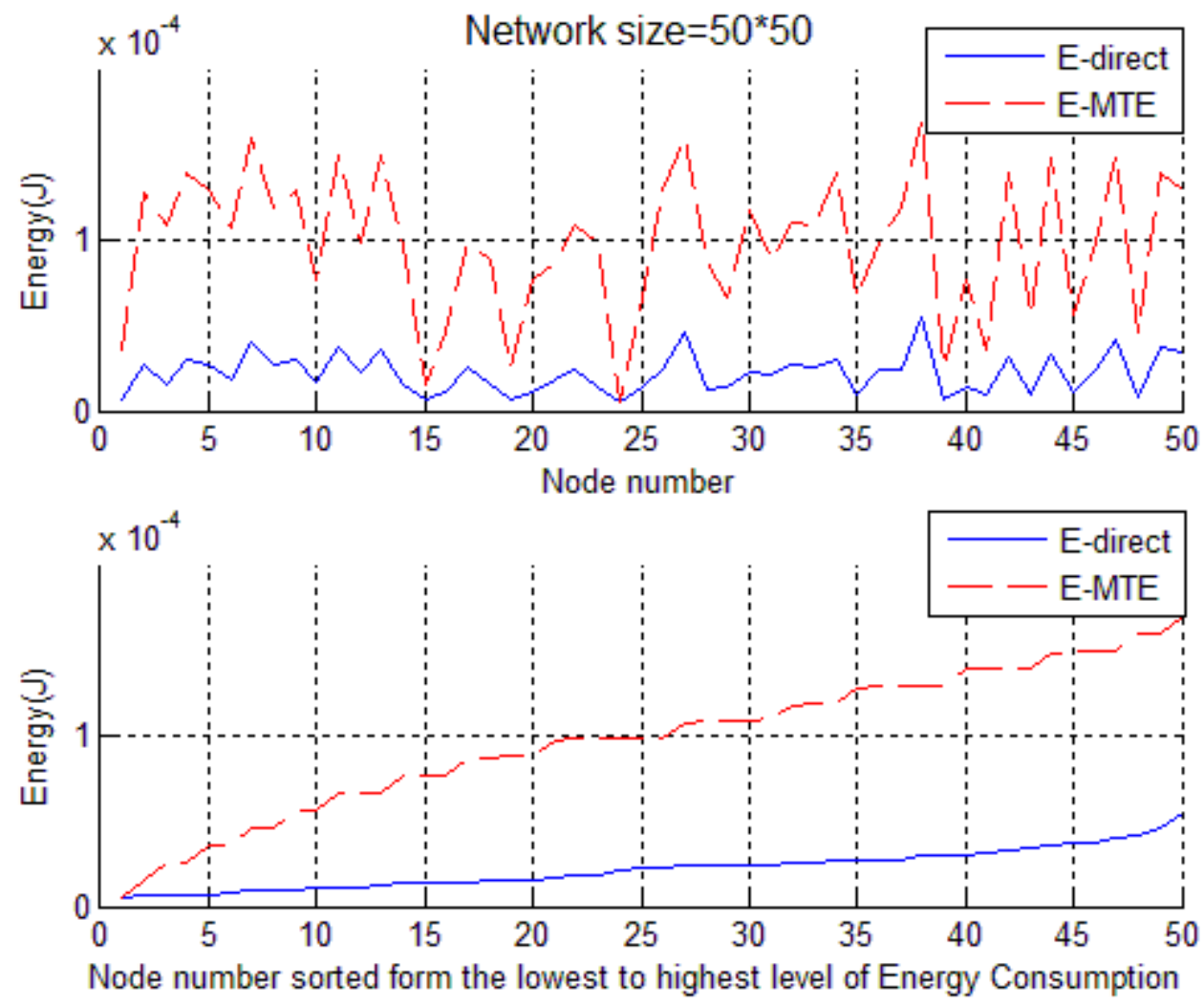

Figure 4.10. Total Energy of the system distributed in $50 \times 50 \mathrm{~m}^{2}$

The graph on the top is showing that in this network size, all the nodes when assumed to be start point, using MTE method consume more energy in comparison to the direct method. 
In the second graph of the Figure 4.10 sensor nodes are sorted from the lowest to highest level of energy consumption. Clearly, for all of the nodes MTE takes the higher place in level of energy consumption. In addition the graph corresponding to $E_{\text {direct }}$ is decreasing slightly, however the graph of $E_{M T E}$ is trending up significantly. From all of the previously mentioned results, it can be concluded, that for this case direct method is more efficient, since the sensor nodes are close to each other.

For the second experiment, size of the network is chosen to be $120 \times 120 \mathrm{~m}^{2}$ and once again 50 number of sensor nodes are distributed through the field. In this network average distance of sensor nodes is approximately $14 \mathrm{~m}$ from each other. Figure 4.12 shows the performance of two methods with unsorted and sorted level of energy respectively. If the network considered to be linear and the sensors are evenly distributed through the area, $d_{\text {critical }}$ is a parameter which is obtained from conditioning $E_{n \text { to } B(\text { direct })}<E_{n \text { to B (MTE) }}$ [24]. Using (4.6) and (4.9) into the above and satisfying the acquired condition will determine the critical distance in which direct transmission is more efficient than the MTE. Then $d_{\text {critical }}$ will be formulated as [24]:

$$
d_{\text {critical }}=\sqrt{\frac{2 E_{\text {elec }}}{n \epsilon_{\text {amp }}}}
$$

This formula can be used when the distance between all of the nodes is equal. In this equation $n$ is the number of nodes in the linear network and for instance is taken to be $n=6$. Substituting $E_{\text {elec }}=50 \mathrm{~nJ} / \mathrm{bit}$ and $\epsilon_{a m p}=100 \mathrm{pJ} / \mathrm{bit} / \mathrm{m}^{2}$ into the equation will result $d_{\text {critical }}=12.90 \mathrm{~m}$. Practically in the network size $120 \times 120 \mathrm{~m}^{2}$ when the number of nodes participating in the MTE routing is equal to 6 , average distance between the nodes is around $13 \mathrm{~m}$. Therefore it can be expected that $120 \mathrm{~m}$ in our experiment, will be very critical size for the network, when one of the direct or 
MTE method wants to be applied as the transmission protocol. Figure 4.11 illustrate the result of network size of $120 \times 120 \mathrm{~m}^{2}$.
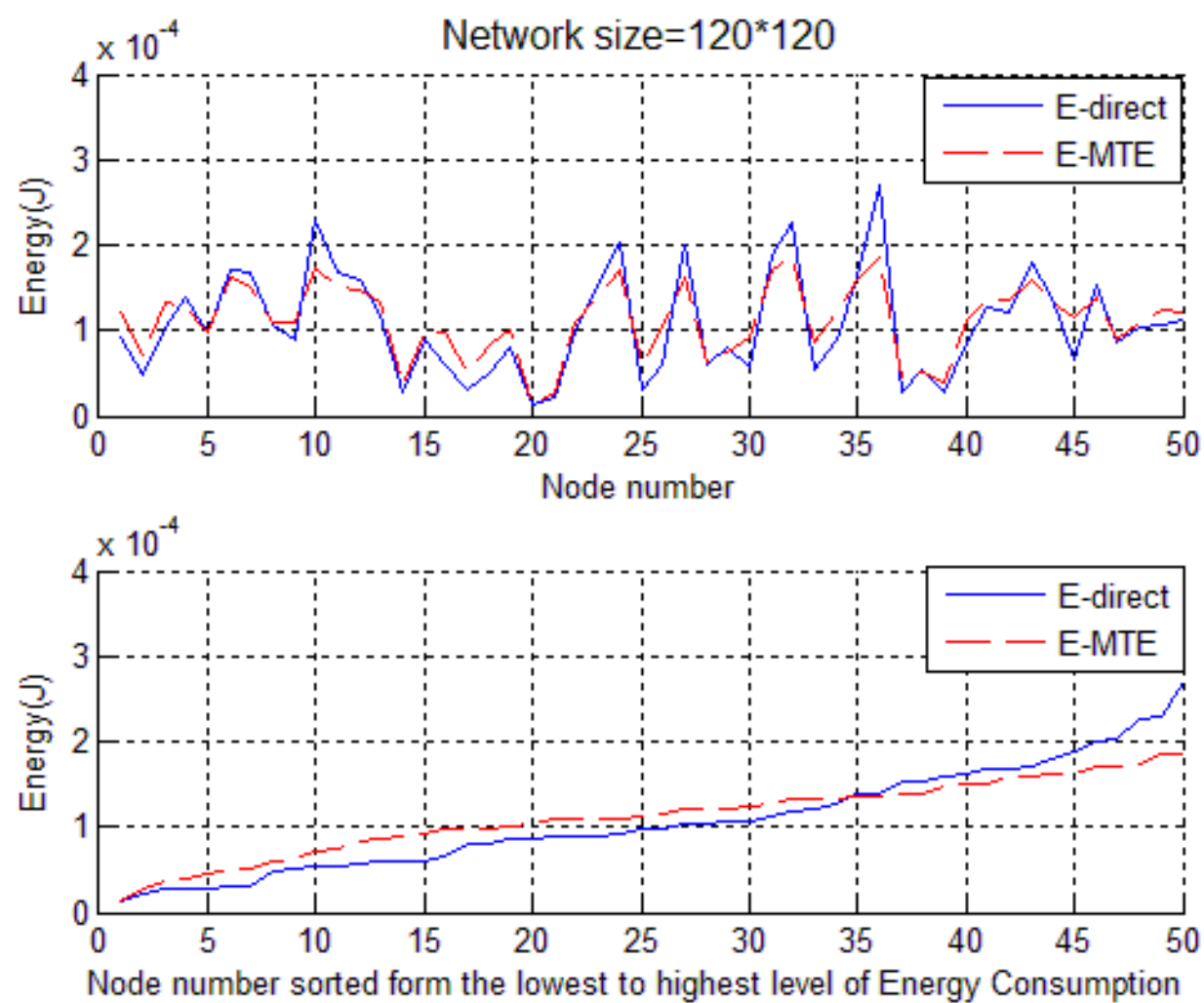

Figure 4.11. Total Energy of the system distributed in $120 \times 120 \mathrm{~m}^{2}$

It was expected in these states, commenting on the efficiency of the both routing protocols will be very critical. It means that, one of these methods should be selected wisely, as the routing protocol of the network. It can be seen that both of direct and MTE methods, have similar performances in many transmission, from source to base station. In this randomized network, direct transmission carried out more efficient results, however it can be expected that, in another simulation with the same conditions, MTE will perform better transmission. 
In the next step, size of the network is set to $160 \times 160 \mathrm{~m}^{2}$ and simulation is carried on again for 50 sensor nodes. Repeating all the stages for this network size, will be conveyed to the graphs, showing in the Figure 4.12:
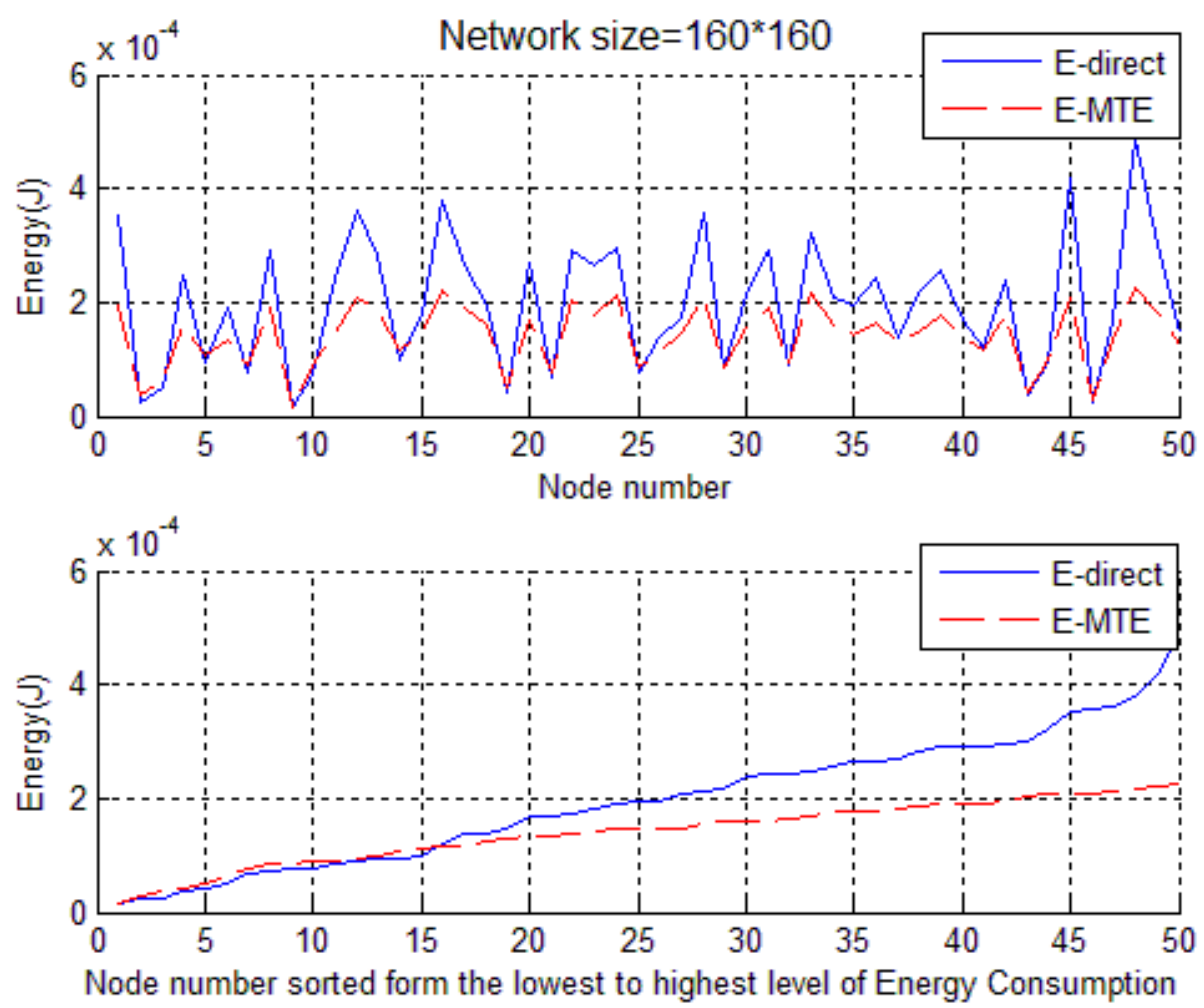

Figure 4.12. Total Energy of the system distributed in $160 \times 160 \mathrm{~m}^{2}$

The average distance of distributed sensor nodes from each other, roughly calculated to $19 \mathrm{~m}$. Considering to the graphs in the Figure 4.12 , clearly proof that, the MTE often has a better performance when is comparing to the direct method. In the second graph which is sorted by the level of energy consumption of each node as a source, the blue line is increasing at the end. These nodes (45 to 50) are probably far away from the base station with the longest distance, that would be near to location $(160,160)$. 
Total amount of energy dissipation in each routing method can be computed, by adding up the energy consumption of each node. Enlargement of the network size was carried out, in three different steps $40 \times 40,120 \times 120$ and $160 \times 160 \mathrm{~m}^{2}$, while other parameters have been kept constant. Now by summarizing total energy dissipation of each method in each step, Table 4.3 will be presented as follows.

Table 4-3: Total Energy dissipation of the system versus network size.

\begin{tabular}{|c|c|c|c|}
\hline Network $\operatorname{size}\left(m^{2}\right)$ & $50 \times 50$ & $120 \times 120$ & $160 \times 160$ \\
\hline$E_{\text {direct }}(J)$ & 0.0012 & 0.0052 & 0.0089 \\
\hline$E_{M T E}(J)$ & 0.0043 & 0.0054 & 0.0069 \\
\hline
\end{tabular}

The results of above table satisfy all assumptions about the behavior of WSNs against of changing size. When direct method is deployed to the network, energy dissipation of the system begins from low amount of $0.0012 \mathrm{~J}$ and goes up to critical value, which is about $0.0052 \mathrm{~J}$ for this randomized network. Then it will grow up instantly up to the value of $0.0089 \mathrm{~J}$ for $160 m \times 160 m$ network size. However, the amount of energy consumption for MTE method is started from $0.0043 \mathrm{~J}$, which is more than direct amount in the same size. After a moderately increase and passing the critical value, it will reach to the value of $0.0069 \mathrm{~J}$ on the top. Aforementioned data simply support the idea that energy consumption of MTE method is increasing at a linear rate in comparison to the direct method. In the upcoming section, the idea will be supported with more details. 


\section{Chapter 5}

\section{HYBRID ENERGY EFFICIENT (HEE) ROUTING PROTOCOL}

Direct and MTE transmission protocols are two popular methods of routing protocol in WSNs. In the previous chapter, efficiency of both protocols in different sizes of the network was analyzed and the results declared that direct method is more energy efficient for the small network size, while MTE has higher quality of performance in the large area.

\subsection{Introducing Hybrid Energy Efficient (HEE) routing protocol}

Considering to the above facts, brings the idea to the mind that how would operate the combination of these two methods? In this research, an energy aware routing protocol based on these two methods will be presented as a new routing protocol and from now, it will be called Hybrid Energy Efficient (HEE) routing protocol.

\subsection{Design and algorithm for Hybrid Energy Efficient (HEE)}

The procedure of calculating energy dissipation of the new routing protocol is started by choosing one of the sensor nodes as a source or starting point. In this stage amount of consumed energy using direct method for transmission is calculated. Then required energy of the MTE method, in order to transmit same packet of data from the same source node to the base station, will be computed. 
So far, required energy of both methods from same source node to the sink is acquired. Therefore, by comparing these two values, that one which is more efficient, can be selected as a desired method of transmission for this specific node.

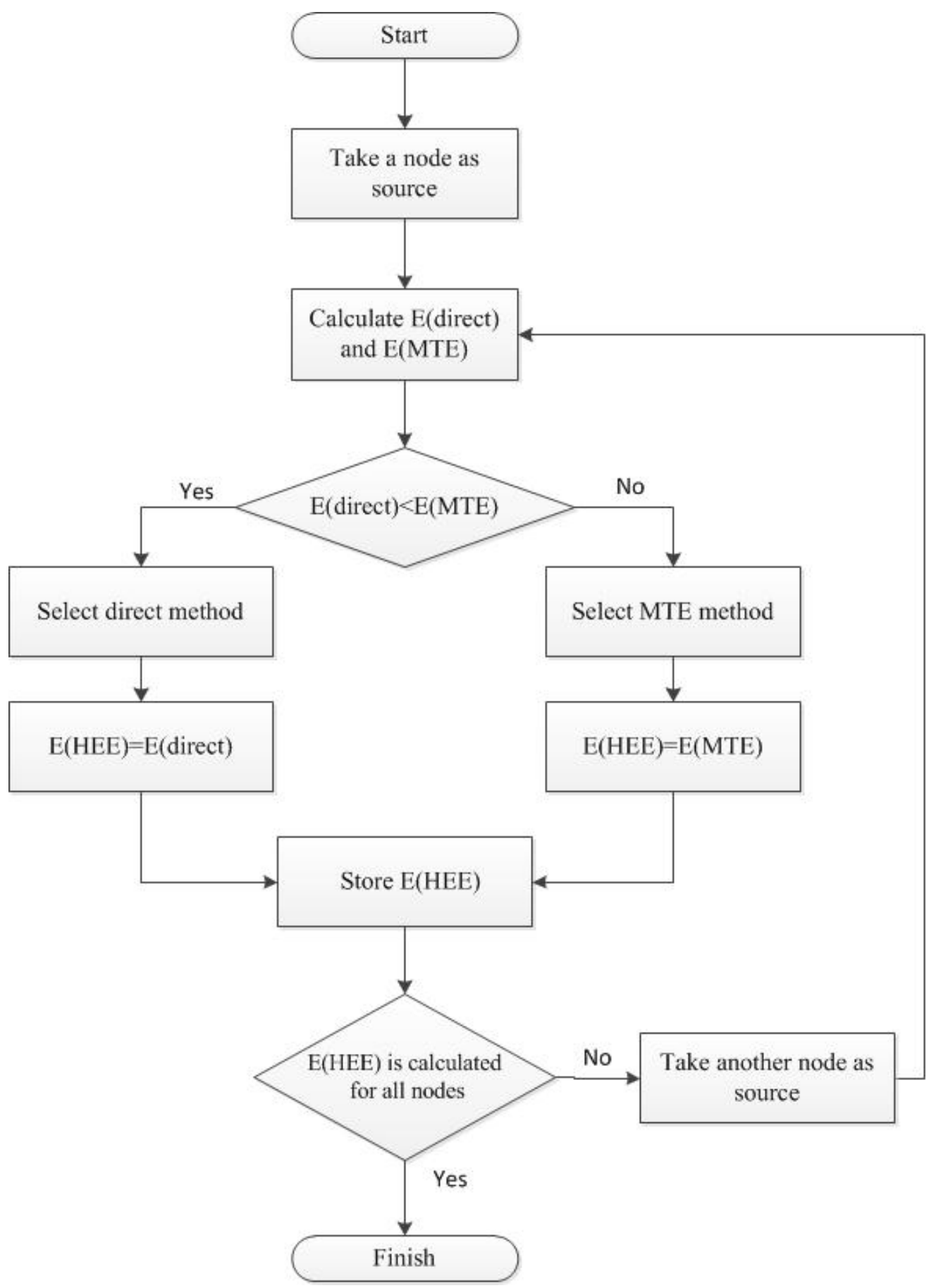

Figure 5.1. Algorithm of Hybrid Energy Efficient (HEE) method 
In addition, it would be the value, which takes place in the HEE method. This procedure can be applied to the entire nodes, as each of them selected to be a source node. It will be continued until HEE values will be calculated for all sensor nodes. Finally, by adding all values of HEE method, total energy expended over the system will be calculated, when the Hybrid energy efficient routing protocol is in use. Figure 5.1 in the above shows the algorithm of HEE, where it is applied to a distributed wireless sensor network.

\subsection{HEE versus Direct and MTE methods}

In this research, routing protocols have been evaluated, mostly by their energy efficiency. In order to be able to comment on HEE performance, same procedure will be applied to HEE as well. At the beginning, performance of HEE will be assessed, when network size and consequently the distance between the nodes is changing. As routine three different side lengths of $(50,120,160 \mathrm{~m})$ are selected, and will be applied to the randomly distributed WSN. In this section for each size, performance of HEE versus direct and MTE will be analyzed simultaneously.

\subsubsection{Network size $50 \times 50$}

Figure 5.2 illustrates the energy dissipation of each 50 nodes trough the $50 \times 50 \mathrm{~m}^{2}$ network area. Required energy of all three methods direct, MTE and HEE are drawn in the blue, red, and green line respectively. Consumed energy of HEE is shown by E-HEE in the legend of the graphs. The point on this graph is that E-direct line is not visible in the picture. The reason is that, in this network size, E-dir and E-HEE lines are match together, and for each node they need same amount of transmission energy. 
Therefore, the green line is plotted entirely on the blue line and then makes it invisible.
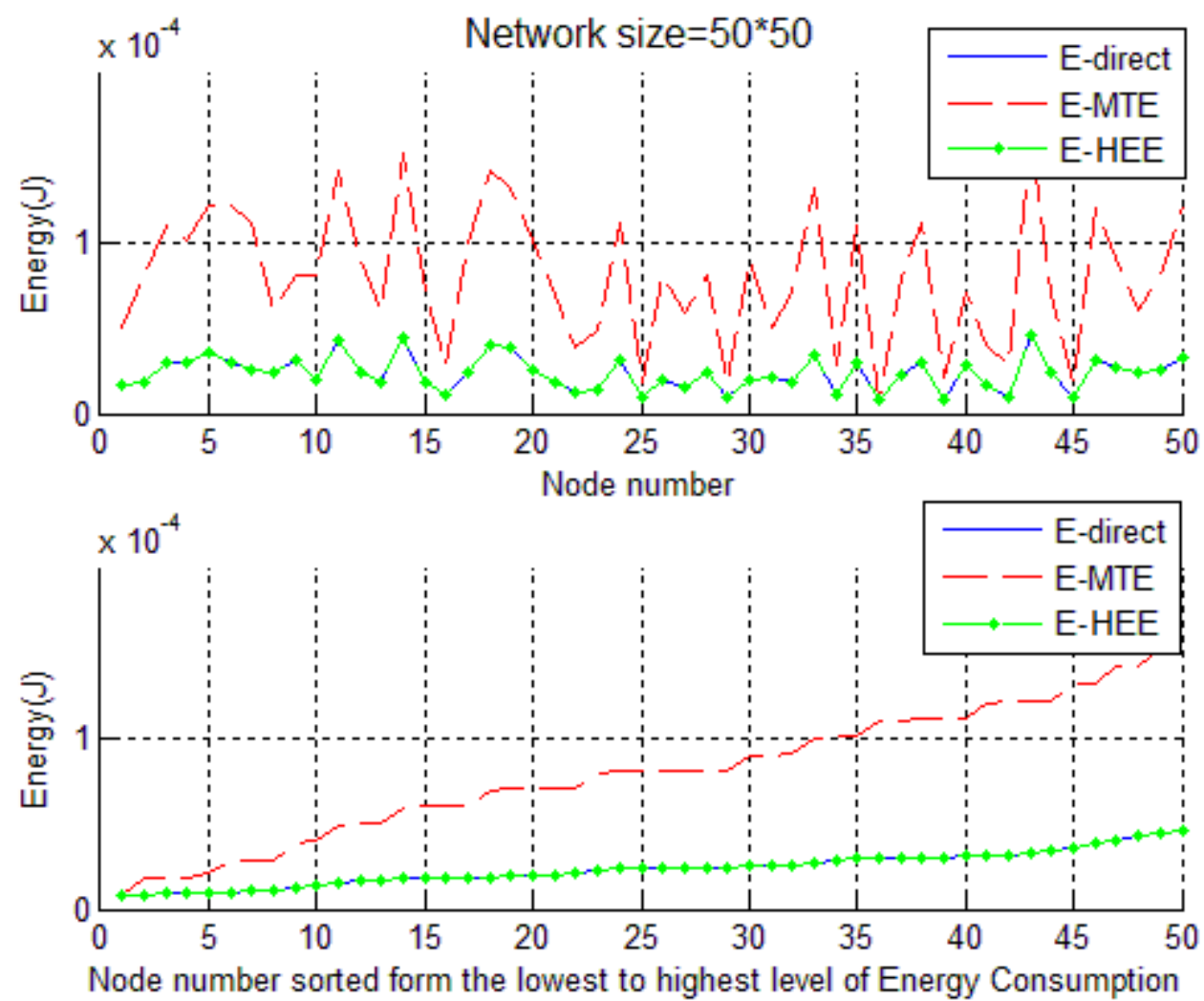

Figure 5.2. Total Energy of the system distributed in 50×50 $\mathrm{m}^{2}$ (HEE versus Direct and MTE)

\subsubsection{Network size $120 \times 120$}

In the next step, critical network size which is $120 m \times 120 m$ is criticized. It is expected that all three method will perform very similar transmission with a view to energy efficiency. Figure 5.3 shows the operation of each of each three routing protocol in the network size of $120 \mathrm{~m} \times 120 \mathrm{~m}$.

From the second graph of the Figure 5.3, which shows the sorted node numbers according to their level of energy consumption, it can be resulted that approximately, in HEE method half of the sensor nodes prefer to transmit data in direct manner and 
the rest, would rather to send data by using MTE algorithm. However all three lines corresponding to E-direct, E-MTE and E-HEE are fluctuating very close to each other, but totally, HEE has more efficient performance in comparison with two other methods.
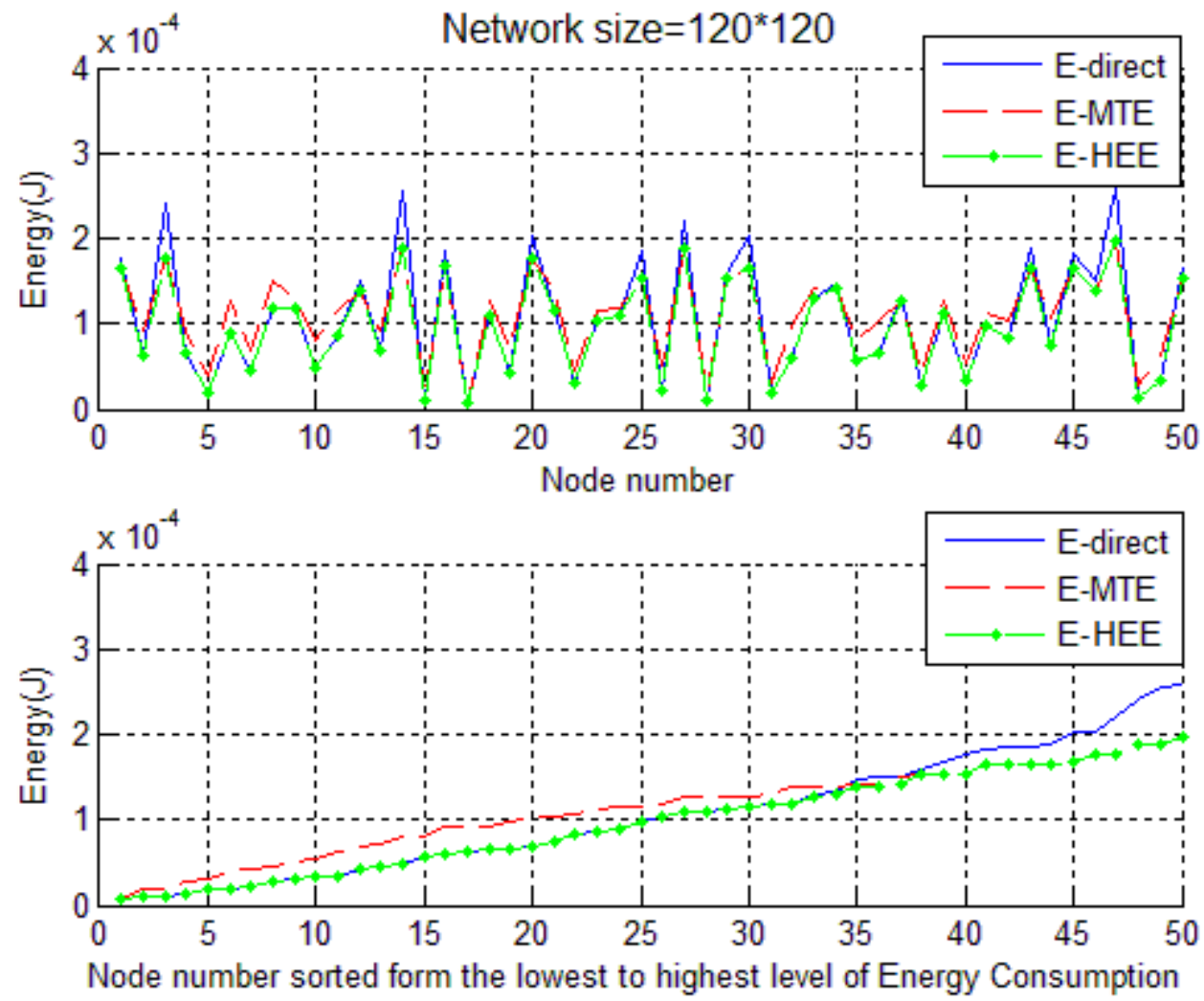

Figure 5.3 Total Energy of the system distributed in $120 \times 120 \mathrm{~m}^{2}$ (HEE versus Direct and MTE)

\subsubsection{Network size $160 \times 160$}

In the last step, once again sensor nodes are distributed over a field of $160 \times 160 \mathrm{~m}^{2}$ and then for the last time in this part, simulation of the network will be carried out. Figure 5.4 shows the graphs obtaining from this simulation.

In this network size $d_{\text {average }}$ is estimated to $19 \mathrm{~m}$, which is not suitable distance for transmitting data directly to the BS. Then majority of the sensors send the data using MTE algorithm. Therefore, clearly HEE has the best performance in this network 
size, while MTE and direct will be ranked respectively in the next places from the energy efficiency point of view.
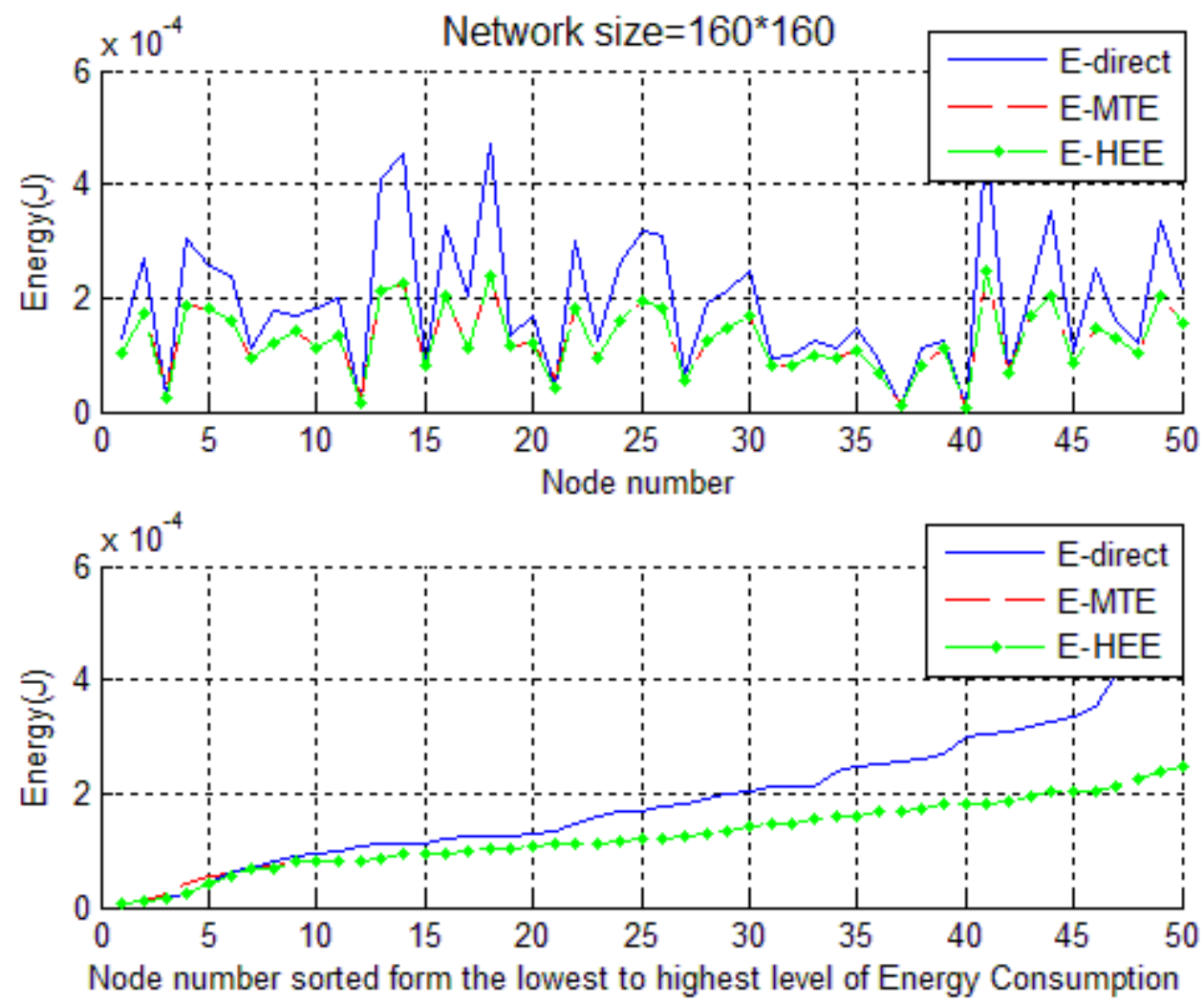

Figure 5.4. Total Energy of the system distributed in 160×160 $\mathrm{m}^{2}$ (HEE versus Direct and MTE)

In each network size, total amount of energy dissipation was recorded and obtained values are presented in Table 5.1. Acquired result for $E_{\text {direct }}$ and $E_{M T E}$ are very close to those of previous simulation, and their behavior is predictable while network size is changing.

Considering to the third row, which is showing the result of HEE method performance, is declaring that in each of the network size, HEE is considerably functioning as the most energy efficient routing protocol. Performance of HEE method will be analyzed in more details and its dependency to the other parameters of WSNs will be criticized in the next section. 
Table 5-1: Total Energy dissipation of the system over different network sizes (HEE versus Direct and MTE)

\begin{tabular}{|c|c|c|c|}
\hline Method & $\mathbf{5 0 \times 5 0}$ & $\mathbf{1 2 0 \times 1 2 0}$ & $\mathbf{1 6 0 \times 1 6 0}$ \\
\hline$E_{\text {direct }}(J)$ & 0.0011 & 0.0056 & 0.0089 \\
\hline$E_{M T E}(J)$ & 0.0040 & 0.0055 & 0.0069 \\
\hline$E_{H E E}(J)$ & 0.0011 & 0.0049 & 0.0061 \\
\hline
\end{tabular}

\subsection{The effect of massage length and Network size on total energy consumption of the system}

It has been mentioned before that, in all simulations, energy for transmitter/receiver electronics and amplifier, have been taken to be constant as: $E_{\text {elec }}=50 \mathrm{~nJ} / \mathrm{bit}$ and $\epsilon_{a m p}=100 \mathrm{pJ} / \mathrm{bit} / \mathrm{m}^{2}$ respectively. Besides length of transmitted massage is considered to be $\mathrm{k}=100 \mathrm{bit}$, while size of the network was varying on three selected sizes. In this section the effect of massage length and network size changing on the energy dissipation will be analyzed, however $E_{\text {elec }}$ and $\epsilon_{\text {amp }}$ are still constant with the same previous values. Analysis will be carried out in three-dimensional spaces (3D), since three parameters of the WSNs are being evaluated in the same time.

Calculation of energy consumption for all sensor nodes in the network was carried out and has been called energy consumption of the system. Now in order to get more precise results, number of bits will be varying from 1 to 100 . On the other hand, network size will be increasing from $1 \times 1$ to $160 \times 160 \mathrm{~m}^{2}$ at the same time. 


\subsubsection{Direct method}

Figure 5.5 illustrates 3D graph of the total energy of the system, where direct method is deployed to the system. It can be seen that number of bits and network size is increasing up to predefined values. Energy consumption is in the minimum level when both of number of nodes and network size are taking the small values. It is clear that it will be maximum, when the number of nodes is close to 100 bit and network size is roughly about $160 \times 160 \mathrm{~m}^{2}$.

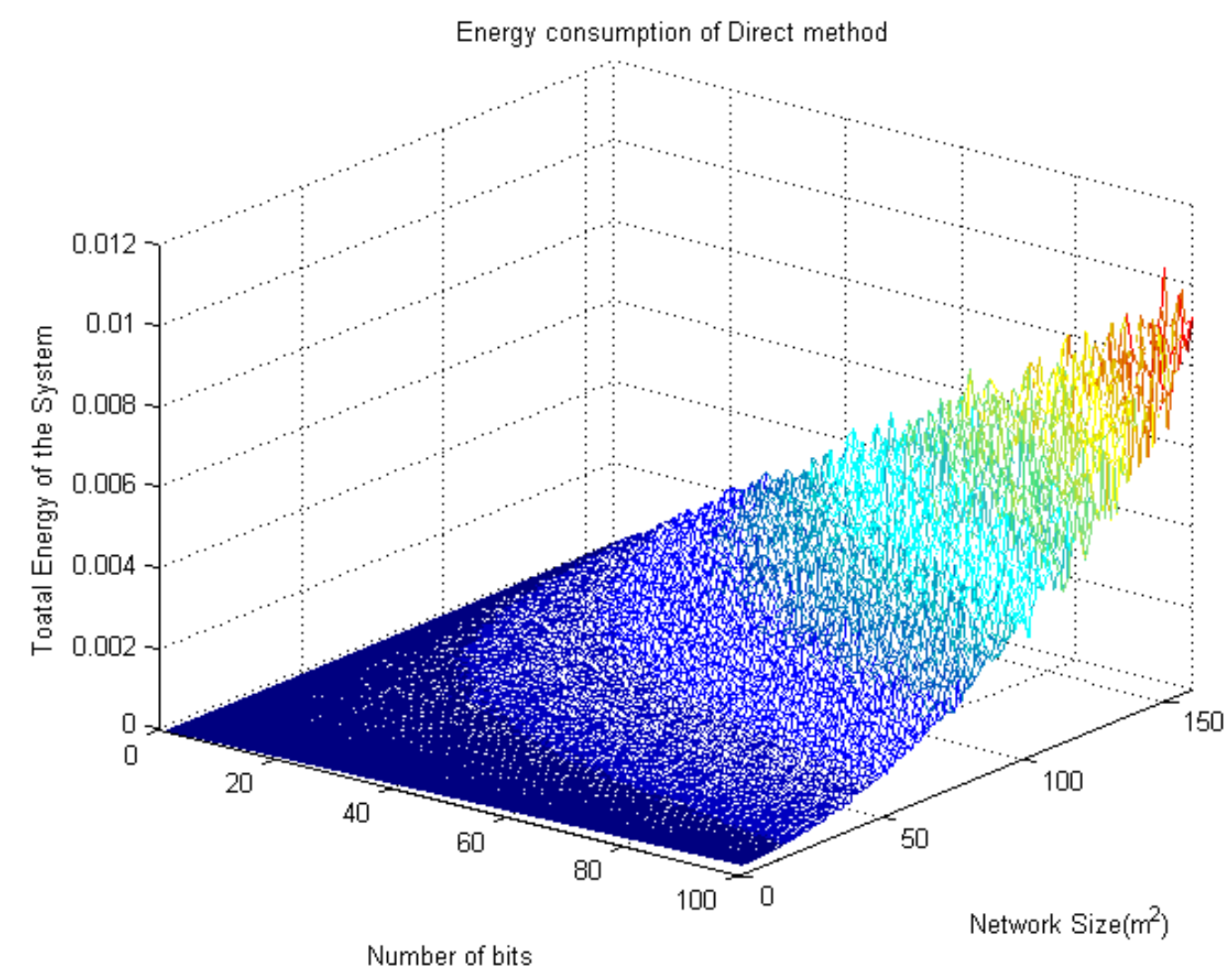

Figure 5.5. Total energy of the system using direct method as network size and number of bits increase

In the shape of this graph, an upward trend was expected, since for the number of bits equal to 100 , total energy of the system was calculated in three network size. There is significant increase when network size is increasing from $50 \times 50 \mathrm{~m}^{2}$ to 
$160 \times 160 \mathrm{~m}^{2}$ and maximum amount of energy consumption is a value close to $0.0090 J$.

\subsubsection{MTE method}

For the next graph, location of 50 sensor nodes in the network is kept unchanged and 3D graph of the number of bits, network size and total energy is sketched, when MTE is responsible to data transmission. The relevant graph is presented in Figure 5.6 .

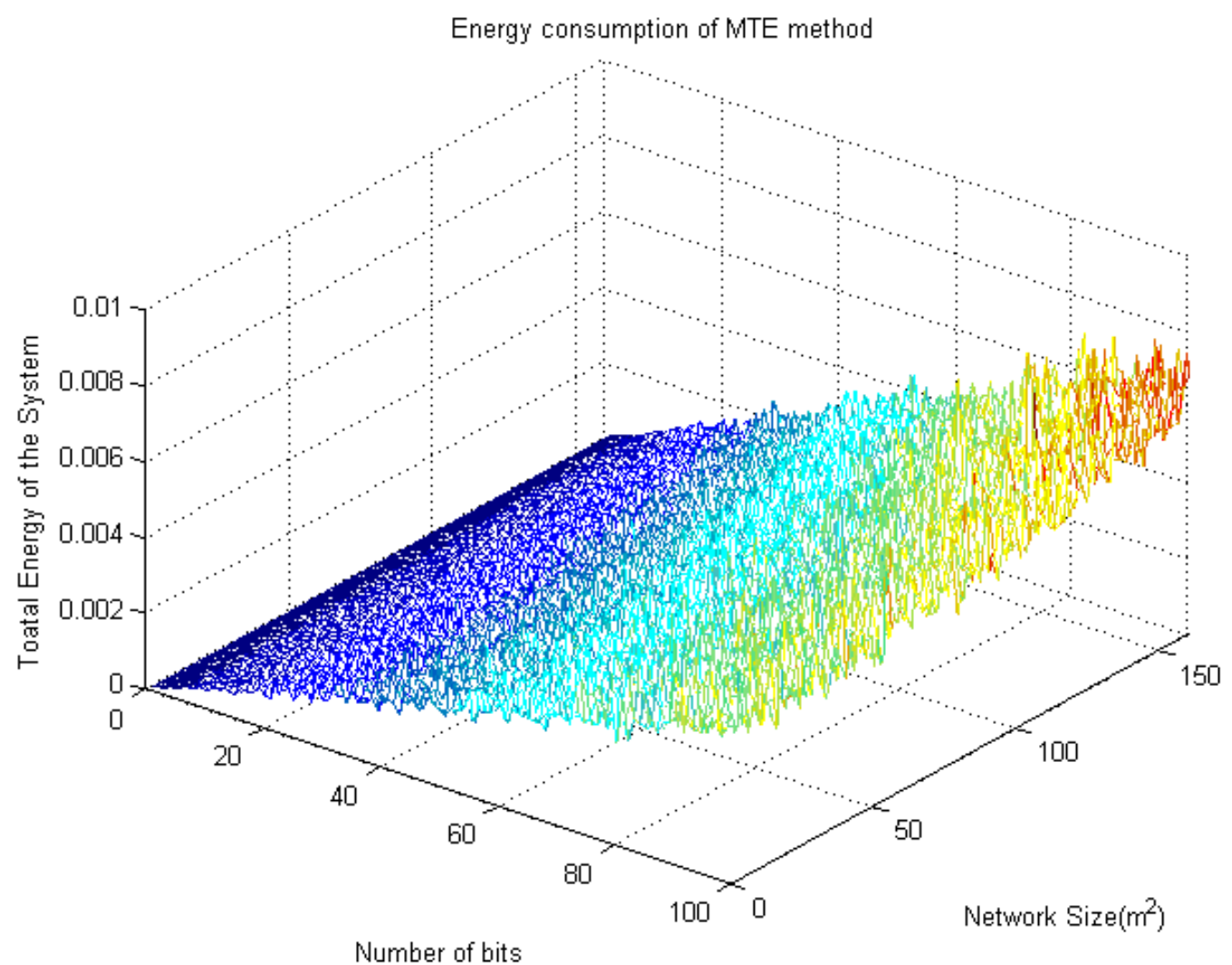

Figure 5.6. Total energy of the system using MTE method as network size and number of bits increase

Considering to the shape of $3 \mathrm{D}$ graph and its ramp, it is obvious that the energy is increasing slightly when the number of nodes and network size are reaching to their biggest values. 3D graph can also proof the results of the previous section. If only variation of the graph for $\mathrm{k}=100$ will be reviewed, it is clear that the amount of total 
energy does not go up too much, which can be seen at the maximum edge of this graph.

Comparing to same edge in the graph of direct energy consumption, MTE method shows more linear behavior when they are dealing with energy consumption.

In addition resulted data in Table 4.4 prove the considerable amount of raising in the direct method, against of insignificant increase in the MTE method while network size is expanding.

\subsubsection{HEE method}

In this section, behavior of the HEE method will be taken into the consideration.

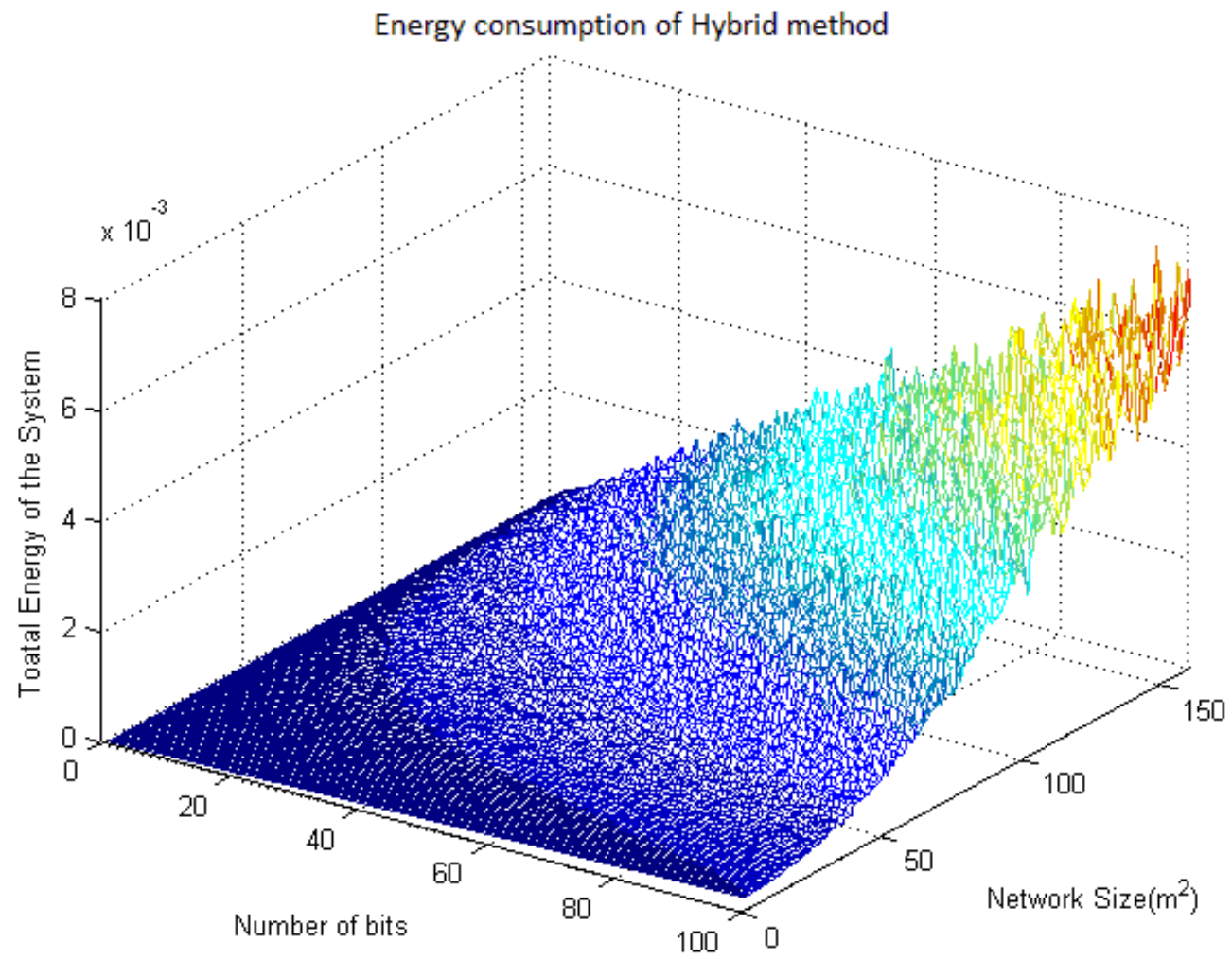

Figure 5.7. Total energy of the system using HEE method as network size and number of bits increase 
Comparing to the direct and MTE methods, it is expected to see less dissipated amount of energy in all positions of the graph, while the number of bits and network size is varying. Moreover maximum amount of energy disipation should be less than the values of the two other methods.

Figure 5.7 illustrates energy consumption of Hybrid energy efficient routing protocol in terms of number of bits and network size. It is is clear that maximum amount of the graph, is less than $0.008 \mathrm{~J}$, which obviously shows more efficient performance than both of direct and MTE method.

Another data analysis, which can be applied to the energy efficiency assessment, is finding the mean of total energy consumption of the system, which is the average value of the plotted 3D graphs. It is one of the most important factors of each protocol, which can represent the efficiency of the method for different sizes and massage length. Summary of all the data from this section is reviewed in Table 5.2.

In the direct method, small values of energy consumption mostly belong to the networks with the small size and short length of massage. While maximum number is too high and makes a great interval between minimum and maximum. This is the characteristic, which makes this method efficient only for small network and short massages. MTE method has better performance in large-scales network in comparison to the direct method.

In Table 5.2, average value of the direct method is more efficient than MTE. This simulation carried out for network size up to $160 \times 160 \mathrm{~m}^{2}$ and maximum $100 \mathrm{bit}$ packet of data. For this condition, direct method achieved better average value. 
Nevertheless, if the network size or massage length becomes greater, MTE method may have a better average value.

Table 5-2: Minimum, Maximum, and average values of direct, MTE and HEE methods

\begin{tabular}{|c|c|c|c|}
\hline Method & Min & Max & Average \\
\hline$E_{\text {direct }}(J)$ & $2.5037 \mathrm{e}-006$ & 0.0098 & 0.0018 \\
\hline$E_{M T E}(J)$ & $3.3481 \mathrm{e}-005$ & 0.0081 & 0.0026 \\
\hline$E_{H E E}(J)$ & $2.5037 \mathrm{e}-006$ & 0.0076 & 0.0014 \\
\hline
\end{tabular}

As HEE method is defined to be energy efficient and combination of these two methods, it has obtained more efficient results in all of the above sections. It means this routing protocol has the capability of being deployed in both of small and large WSNs in terms of network size and length of massage.

\subsection{Performance analysis of HEE method}

HEE method performs the most energy efficient data transmission in both of small and large network sizes. Deploying an energy efficient protocol increases the network lifetime, since it uses less energy for each transmission. It means this method of transmission is suitable for WSNs, when we are dealing with the networks, which need to be in use for a long time. Moreover, this method can be used in the networks with variable sizes, massage length and node numbers. As an example, it can be imagined that for environmental monitoring, there is sensor field in which the area needs to be monitored is varying in different seasons. It means the network size will be changed in different times. Furthermore, the length of massage is not constant, while the collected information from the environment is not fixed. In these situations for many cases, replacing the batteries of the spread out sensor nodes is very difficult, when one of them is discharged or is going to be died. Therefore, 
increasing the network lifetime as much as possible is one of the challenges. In these conditions, HEE is the desired routing protocol, since it copes with the required criteria.

Efficiency of HEE method can also be measured in terms of execution time and required memory. These are important parameters in complexity analysis of the system. Execution time is significantly important in the real-time applications in which response time is the key point. For instance military and security alarm are two applications of WSNs in which delay in reporting an event is not desired. Hence, the routing protocol for these applications must be selected according to their requirements. Execution time of HEE is greater than direct and MTE methods since, in order to find the HEE value for each node, both of direct and MTE values are needed. Therefore, it takes more time and it should be considered that how much is the difference between HEE's execution time and other methods.

Moreover, in term of required memory, HEE has its own constraints. Calculating both of direct and MTE values and storing HEE value needs more memory in comparison to each of two basic methods. Therefore, required memory is also one of the main challenges for this protocol, which may cause some limitations for its deployment into the wireless sensor networks.

\subsubsection{Computational Complexity of HEE}

Execution time of method mostly estimated by counting the number of elementary operations, performed by their relevant algorithm and it can be referred as computational complexity of the algorithm. The algorithm used to perform HEE protocol, is based on direct and MTE algorithms. Therefore, computational 
complexity of HEE can be compared to these two basic methods and the result shows the number of basic mathematical operations, which is used in each of three methods. Table 5-3 shows the number of addition, subtraction, multiplication, division and comparison that is used in the algorithm of Direct, MTE and HEE methods.

Table 5-3: Computational complexity of Direct, MTE and HEE Routing protocols

\begin{tabular}{|c|c|c|c|c|}
\hline Method & $\begin{array}{c}\text { Number of } \\
\text { Addition/ } \\
\text { Subtraction }\end{array}$ & $\begin{array}{c}\text { Number of } \\
\text { Multiplication/ } \\
\text { Division }\end{array}$ & $\begin{array}{c}\text { Number of } \\
\text { Square root/ } \\
\text { Exponentiation }\end{array}$ & $\begin{array}{c}\text { Number of } \\
\text { Comparison }\end{array}$ \\
\hline Direct & 3 & 4 & 6 & - \\
\hline$M T E$ & $5+6 n$ & 9 & $6+11 n$ & 4 \\
\hline$H E E$ & $10+6 n$ & 13 & $10+11 n$ & 5 \\
\hline
\end{tabular}

This table is calculated for one singe node, transmitting its data to the base station. In direct transmission method, there is no intermediate node, and source node sends its data directly to the sink node. Therefore, number of needed operation in this algorithm is constant for all of the nodes in the network. While in MTE and perhaps HEE data is transferred via the intermediate nodes. The parameter $n$ shows the number of intermediate nodes in order to form MTE pathway. Consequently, number of mathematical operations for these two methods depends on the number of nodes, which are forming the path.

Number of comparison in this table, refers to the number of if and while commands, which were used to simulate the relevant algorithm. There is no comparison command for direct method, while the number of comparison for MTE and HEE is 4 and 5 respectively. 
This table is more useful where the amount of taken time for each of the basic operation is known individually. In this case, time complexity of each algorithm can be estimated by simple calculations.

Direct transmission uses simple algorithm for sending data to the BS. Therefore, time execution of this method is very short. MTE has some complexities for data transmission and its execution time would be more than direct method. The different between execution time of HEE and MTE, is approximately the execution time of direct transmission per each node, since HEE is the combination of these two methods. Therefore, the greater amount of taken time for HEE in comparison to MTE, can be negligible in some of the applications of wireless sensor networks. 


\section{Chapter 6}

\section{CONCLUSIONS AND FUTURE WORK}

In this thesis, a new energy efficient routing protocol for wireless sensor network has been presented. This routing protocol is based on two popular routing protocols of WSNs, namely direct and minimum energy transmission (MTE) methods. On the other hand, wireless sensor networks are very resource constrained, since they have limited source of energy. Hence, in the design of new method, energy efficiency of this routing protocol has been considered as one of the important characteristics.

Therefore, it has been named Hybrid energy efficient (HEE) routing protocol. For this purpose, an algorithm for HEE is designed and then, proposed routing protocol has been implemented, using the HEE algorithm. Therefore, HEE method has been simulated, by deploying the proposed method in a randomly distributed WSN. The simulation results can be summarized by the following facts.

1) The proposed method (HEE) has the appropriate performance in both of large and small size of the networks, since comparing to direct and MTE methods, HEE gains more energy efficient performance in any network size.

2) HEE also shows efficient result in term of energy consumption, when the massage length is changing.

3) Calculating mean and maximum values of the consumed energy for the same network, when each of direct, MTE and HEE methods are deployed, 
demonstrates that HEE routing protocol consumes less energy in both parameters.

\subsection{Future Work}

HEE is more energy efficient than direct and MTE, while it uses both of these two methods for data transmission. For each node only once, direct and MTE energy consumption is calculated and takes place as HEE. As a future work, HEE can be more energy efficient by making it Intelligent Hybrid Energy Efficient (IHEE).

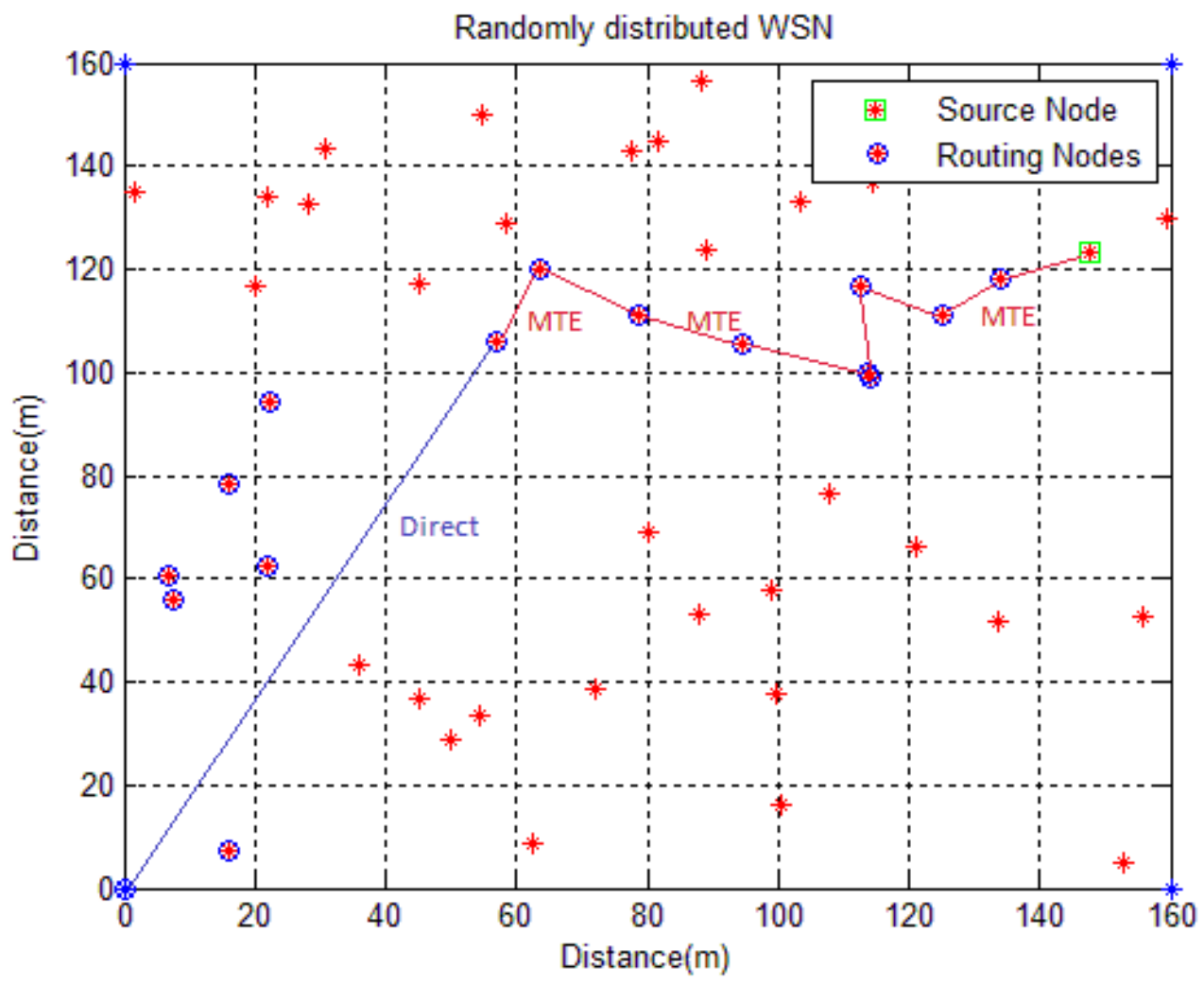

Figure 6.1. Data transmission for one random sensor node using IHEE method

Data transmission for one node can be carried out using both of direct and MTE. It means each time that a source sensor node is in the sending mode, firstly required energy for direct and MTE is calculated. If the direct energy consumption is less than MTE, sensor node decides to send data directly. Otherwise, data is transmitted to its 
nearest neighbor, using MTE algorithm. For this neighbor, which is now in charge of data transmission, both of direct and MTE energy consumption is computed again. Even in this stage, if direct transmission becomes more efficient, this sensor sends its data immediately by direct method. Otherwise, this procedure will be continued until that either one node sends the data directly or MTE completes its algorithm path to the base station. Figure 6.1 shows that IHEE constructs the MTE path, and the path is followed until that MTE is more efficient. As soon as direct method consumes less energy than MTE, data will be sent directly to the base station. 


\section{REFERENCES}

[1] I. F. Akyildiz, W. Su, Y. Sankarasubramaniam E. Capirci, "Wireless sensor networks: a survey”, Computer Networks, vol 38, no 4, Mar. 2002.

[2] C. Alippi, G. Anastasi, M. Di Francesco, M. Roveri, "Energy management in wireless sensor networks with energy-hungry sensors", IEEE Instrumentation and Measurement Magazine, vol. 12, pp. 16-23, Apr. 2009.

[3] V. C. Gungor, and G. P. Hancke, "Industrial wireless sensor networks: challenges, design principles, and technical approaches", IEEE Transactions on Industrial Electronics, vol. 56, no. 10, Oct. 2009.

[4] IML Group Plc, "Wireless hart specification released", Control Engineering Europe, vol. 8, no. 3, pp. 8-9, Jul. 2007.

[5] A. Perrig, J. Stankovich, and D. Wagner, "Security in wireless sensor networks", Commun ACM, vol. 47, no. 6, pp. 53-57, Jun. 2004.

[6] N. Aakvaag, M. Mathiesen, and G. Thonet, "Timing and power issues in wireless sensor networks-an industrial test case" in Proc. Int. Conf. Parallel Process Workshops, pp. 419-426, 2005. 
[7] D. Estrin, L. Girod, G. Pottie, M. Srivastava, "Instrumenting the world with wireless sensor networks", Proc. Acoustics, Speech, and Signal Processing, vol. 4, pp. 2033-2036, 2001.

[8] M. Kahvakka, J. Suhonen, P. Hamalainen, M. Hannkainen, Hamalainen, "Ultralow energy wireless sensor networks in practice”, John Wiley, 2007.

[9] S. Singh, M. P. Singh, and D. K. Singh, "Routing protocols in wireless sensor networks - A survey" International Journal of Computer Science and Engineering Survey (IJCSES) vol.1, No.2, Nov. 2010.

[10] Y. Xu, J. Heidemann, and D. Estrin, "Geography-informed energy conservation for ad-hoc routing", Proceedings ACM/IEEE MobiCom'01, Rome, Italy, pp. 70-84, Jul. 2001.

[11] Y. Yu, R. Govindan, and D. Estrin, "Geographical and energy aware routing: A recursive data dissemination protocol for wireless sensor networks", Technical Report UCLA/CSD-TR-01-0023, UCLA Computer Science Department, May 2001.

[12] V. Rodoplu and T. H. Meng, "Minimum energy mobile wireless networks", IEEE Journal on Selected Areas in Communications, vol. 17, no. 8, pp. 1333-1344, Aug. 1999. 
[13] A. Boukerche, X. Cheng, and J. Linus, "Energy-aware data-centric routing in microsensor networks", Proceedings ACM MSWiM, in conjunction with ACM MobiCom, San Diego, CA, pp. 42-49, Sept. 2003.

[14] C. Intanagonwiwat, R. Govindan, and D. Estrin, "Directed diffusion: A scalable and robust communication paradigm for sensor networks", Proceedings ACM MobiCom'00, Boston, MA, pp. 56-67, Aug. 2000.

[15] S.K. Singh, M.P. Singh, and D.K. Singh, "A survey of energy-efficient hierarchical cluster-based routing in wireless sensor networks", International Journal of Advanced Networking and Application (IJANA), vol. 02, issue 02, pp. 570-580, Sept.-Oct. 2010.

[16] W. R. Heinzelman, A. Chandrakasan, and H. Balakrishnan, "Energy-efficient communication protocol for wireless microsensor networks", in IEEE Computer Society Proceedings of the Thirty Third Hawaii International Conference on System Sciences (HICSS '00), Washington DC, USA, vol. 8, pp. 8020, Jan. 2000.

[17] S. Lindsey and C.S. Raghavendra, "PEGASIS: Power-efficient Gathering in Sensor Information System”, Proceedings IEEE Aerospace Conference, vol. 3, Big Sky, MT, pp. 1125-1130, Mar. 2002.

[18] O. Younis and S. Fahmy, "Heed: A hybrid, Energy-efficient, Distributed clustering approach for ad-hoc networks", IEEE Transactions on Mobile Computing, vol. 3, no. 4, pp. 366-369, Oct.-Dec. 2004. 
[19] B. Karp and H. T. Kung, "GPSR: Greedy perimeter stateless routing for wireless networks", Proceedings ACM MobiCom'00, Boston, MA, pp. 243-254, Aug. 2000.

[20] W. Chang, G. Cao, and T. La Porta, "Dynamic proxy tree-based data dissemination schemes for wireless sensor networks", Proceedings IEEE MASS'04, Fort Lauderdale, FL, pp. 21-30, Oct. 2004.

[21] S. Lindsey, C. S. Raghavendra, and K. M. Sivalingam, "Data gathering in sensor networks using the energy delay metric", Proceedings IPDPS'01, San Francisco, CA, pp. 2001-2008, Apr. 2001.

[22] X. Du and F. Lin, "Improving routing in sensor networks with heterogeneous sensor nodes", Proceedings IEEE VTC'05, Dallas, TX, pp. 25282532, Sept. 2005.

[23] K. Akkaya and M. Younis, “An energy-aware QoS routing protocol for wireless sensor networks" in the Proceedings of the IEEE Workshop on Mobile and Wireless Networks (MWN 2003), Providence, Rhode Island, May 2003.

[24] R. Abrishambaf, S. N. Bayindir and M. Hashemipour, "Energy analysis of routing protocols in wireless sensor networks for industrial applications", Journal of Systems and Control Engineering, Nov. 2011. 
[25] Z. Rezaei, S. Mobininejad, "Energy Saving in Wireless Sensor Networks", International Journal of Computer Science \& Engineering Survey (IJCSES), vol.3, No.1, Feb. 2012.

[26] T. Rappaport, "Wireless communication: principles and practice", New Jersey, Prentice-Hall, 1996. 